\title{
Nonlinear Disturbance Observer-Based Adaptive Sliding Mode Control for a Generic Hypersonic Vehicle
}

\author{
Jianguo Guo $\mathbb{D}$, Hao Zhang $\mathbb{D}^{D}$, Xiaodong $L u(\mathbb{D}$, and Jun Zhou $\mathbb{D}$ \\ Institute of Precision Guidance and Control, Northwestern Polytechnical University, Xi'an 710072, China \\ Correspondence should be addressed to Jianguo Guo; guojianguo@nwpu.edu.cn
}

Received 21 May 2017; Accepted 9 November 2017; Published 21 February 2018

Academic Editor: Christopher J. Damaren

Copyright (C) 2018 Jianguo Guo et al. This is an open access article distributed under the Creative Commons Attribution License, which permits unrestricted use, distribution, and reproduction in any medium, provided the original work is properly cited.

\begin{abstract}
In this paper, a new adaptive sliding mode control method is presented for the longitudinal model of a generic hypersonic vehicle subject to uncertainties and external disturbance. Firstly, an oriented-control model with mismatched uncertainties is built for a generic hypersonic vehicle. Secondly, the back-stepping technique is introduced to design a sliding mode controller with an adaptive law to adapt to the disturbance and uncertainty. Thirdly, a set of nonlinear disturbance observers are designed to estimate the lumped disturbance and compensate the sliding mode controller, and the stability of the proposed controller is analyzed by utilizing Lyapunov stability theory. Finally, simulation results show that the effectiveness of the proposed controller is validated by the nonlinear model and the proposed method exhibits promising robustness to mismatched uncertainties.
\end{abstract}

\section{Introduction}

Generic hypersonic vehicles (GHVs) provide a reliable way to enter space and attract worldwide attentions in recent years. As GHVs are sensitive to physical and aerodynamic parameter changes, a concernful task is to design an efficient control system that makes the flight of GHVs feasible.

Faced with the complexities of GHV dynamics, the design methods of the guidance and control system have attracted considerable interests [1-3]. However, modeling inaccuracies and various disturbance can lead to some adverse effects on the controller; it is a challenging problem to design the controller of GHVs [4].

Sliding mode control (SMC) is widely used in dealing with parameter uncertainties and external disturbances for the flight of GHVs [5-8]. Furthermore, back-stepping technique is also an effective way for the control system design, in which the virtual control input can be obtained at each step and the actual controller comes to being [9-12]. The combination of back-stepping method and dynamic surface method is used to design a robust controller. However, the problem of compute explosion in the back-stepping method have to be solved
Meanwhile, the adaptive control approach is applied to adapt to the parameter uncertainties as well as constraint on states and control inputs [12-20]. By selecting a proper adaptive law, the satisfied performance can be easily achieved.

It is well known that the disturbance observer is an efficient and active method to compensate the controller against uncertainties and external disturbances [21-24]. At present, the nonlinear disturbance observer (NDO) can be employed to design the controller of the GHV with matched disturbance and mismatched disturbance [24-29].

Motivated by the abovementioned researches, a new adaptive SMC strategy that consists of the adaptive control method, back-stepping method, and nonlinear disturbance observer method is proposed in this paper. In the proposed controller framework, a new nonlinear disturbance observer (NDO) is employed to estimate the lumped disturbances that are introduced into the sliding surface and virtual control input at each step to compensate the effects of disturbances. It is proved that the closed-loop system is asymptotically stable here. Finally, simulation results show that the proposed method has a good disturbance rejection performance without sacrificing the nominal control performance. 
The key innovations are listed below:

(i) A new adaptive SMC method is proposed to meet the flight performance for the GHV with highly nonlinear and mismatched uncertainties.

(ii) A nonlinear disturbance observer is introduced into the control system to estimate the lumped uncertainties and external disturbance to compensate the sliding mode controller.

(iii) The compute explosion problem is solved in the back-stepping method by utilizing the adaptive controller.

\section{Hypersonic Air Vehicle Model}

2.1. Original Model. The longitudinal dynamics of a GHV can be described with a set of differential equations composed by velocity $V$ and the flight path angle $\gamma$, altitude $h$, angle of attack $\alpha$, and pitch rate $q[3]$.

$$
\begin{aligned}
& \dot{V}=\frac{T \cos \alpha-D}{m}-\frac{\mu \sin \gamma}{r^{2}}+d_{1}, \\
& \dot{\gamma}=\frac{L+T \sin \alpha}{m V}-\frac{\mu-V^{2} r}{V r^{2}} \cos \gamma+d_{2}, \\
& \dot{h}=V \sin \gamma, \\
& \dot{\alpha}=q-\dot{\gamma}+d_{3}, \\
& \dot{q}=\frac{M_{y y}}{I_{y y}}+d_{4},
\end{aligned}
$$

where $d_{i}(i=1,2,3,4)$ represents the lumped disturbances in (1), (2), (4), and (5), respectively. $m, I_{y y}$, and $\mu$ represent the mass of the vehicle, moment of inertia, and gravity constant, respectively. $L, D, T$, and $M_{y y}$ represent the lift force, the drag force, the thrust force, and the pitching moment, respectively. The $r$ is the radial distance from Earth's center. They can be described as

$$
\begin{aligned}
L & =\frac{1}{2} \rho V^{2} S C_{L}, \\
T & =\frac{1}{2} \rho V^{2} S C_{T}, \\
D & =\frac{1}{2} \rho V^{2} S C_{D}, \\
M_{y y} & =\frac{1}{2} \rho V^{2} S \bar{c}\left[C_{M}(\alpha)+C_{M}\left(\delta_{e}\right)+C_{M}(q)\right], \\
r & =h+R_{\mathrm{E}},
\end{aligned}
$$

where $C_{L}, C_{T}$, and $C_{D}$ represent the lift, thrust, and drag coefficients, respectively. $C_{M}(\alpha), C_{M}(q)$, and $C_{M}\left(\delta_{e}\right)$ represent the coefficients referred to as the angle of attack, pitch rate, and elevator deflection, respectively. The parameters $\rho, S, \bar{c}$, and $R_{\mathrm{E}}$ represent the air density, the reference area, the mean aerodynamic chord, and the radius of the earth, respectively.
The engine dynamics is modeled by a second-order system

$$
\ddot{\beta}=-2 \zeta \omega_{n} \dot{\beta}-\omega_{n}^{2} \beta_{c}
$$

where $\beta$ is the throttle setting, and $\beta_{c}$ is the throttle setting command.

In this paper, the aerodynamics and physical coefficients are simplified around the nominal cruising flight. The terms of $\Delta$ denote the parameter uncertainties.

$$
\begin{aligned}
C_{L} & =0.6203 \alpha, \\
C_{D} & =0.6450 \alpha^{2}+0.0043378 \alpha+0.003772, \\
C_{T} & = \begin{cases}0.02576 \beta, & \text { if } \beta<1, \\
0.0224+0.00336 \beta, & \text { if } \beta>1,\end{cases} \\
C_{M}(\alpha) & =-0.035 \alpha^{2}+0.036617 \times\left(1+\Delta C_{m \alpha}\right) \alpha+5.3261 \times 10^{-6}, \\
C_{M}(q) & =\left(\frac{\bar{c}}{2 V}\right) q\left(-6.796 \alpha^{2}+0.3015 \alpha-0.2289\right), \\
C_{M}\left(\delta_{e}\right) & =c_{e}\left(\delta_{e}-\alpha\right), \\
m & =m_{0}(1+\Delta m), \\
I_{y y} & =I_{0}(1+\Delta I), \\
S & =S_{0}(1+\Delta S), \\
\bar{c} & =\bar{c}_{0}(1+\Delta \bar{c}), \\
\rho & =\rho_{0}(1+\Delta \rho), \\
c_{e} & =0.0292\left(1+\Delta c_{e}\right),
\end{aligned}
$$

where $m_{0}=9375, \quad I_{0}=7 \times 10^{6}, \quad S_{0}=3603, \quad \bar{c}=80, \quad$ and $\rho_{0}=0.24325 \times 10^{-4}$. The maximum value of the additive uncertainties is listed below.

$$
\begin{gathered}
|\Delta m| \leq \Delta_{m}, \\
|\Delta I| \leq \Delta_{I}, \\
|\Delta S| \leq \Delta_{S}, \\
|\Delta \overline{\bar{c}}| \leq \Delta_{\bar{c}}, \\
|\Delta \rho| \leq \Delta_{\rho}, \\
\left|\Delta c_{\mathrm{e}}\right| \leq \Delta_{c_{e}}, \\
\left|\Delta c_{M \alpha}\right| \leq \Delta_{c_{M \alpha}} .
\end{gathered}
$$

The velocity is mainly related to throttle setting $\beta$ while the change of altitude is mainly related to the elevator deflection $\delta_{e}$.

\subsection{Preparation and System Transformation}

2.2.1. Preparation. The model of a GHV described by (1), (2), (3), (4), and (5) can be decoupled into two parts, which are velocity subsystem and altitude subsystem. In this paper, the flight path angle is set in a small area. 
Assumption 1. The lumped disturbances are bounded and the maximum value is as follows:

$$
\left|d_{i}\right| \leq \xi_{i}, \quad i=1,2,3,4,
$$

where $d_{i}$ represents the lumped disturbances in (1), (2), (4), and (5), respectively. $\xi_{i}>0$ is a known constant.

2.2.2. System Transformation. The variable states are chosen as $x_{1}=V, x_{2}=\gamma, x_{3}=\theta$, and $x_{4}=q$, where $\theta=\alpha+\gamma$.

(1) Velocity subsystem:

$$
\begin{aligned}
& \dot{x}_{1}=f_{1}+g_{1} u_{1}+d_{1}, \\
& u_{1}=\beta, \\
& y_{1}=x_{1} .
\end{aligned}
$$

If $\beta>1$,

$f_{1}=\frac{\mu \sin x_{3}}{r^{2}}-\frac{D}{m}+\frac{1}{2} \rho x_{1}^{2} S \times 0.0224 \frac{\cos \left(x_{4}-x_{3}\right)}{m}$,

$g_{1}=\frac{1}{2} \rho x_{1}^{2} S \times 0.00336 \frac{\cos \left(x_{4}-x_{3}\right)}{m}$.

Otherwise

$$
\begin{aligned}
& f_{1}=-\frac{\mu \sin x_{3}}{r^{2}}-\frac{D}{m}, \\
& g_{1}=\frac{1}{2} \rho x_{1}^{2} S \times 0.02576 \frac{\cos \left(x_{4}-x_{3}\right)}{m} .
\end{aligned}
$$

(2) Altitude subsystem: the tracking error is described by $\tilde{h}=h-h_{d}$. The altitude is denoted by $x_{2}$ as well as the altitude command is represented by $x_{2 d}$. Then, the derivative of altitude tracking error can be obtained as

$$
\dot{\tilde{h}}=\dot{h}-\dot{h}_{d}=x_{1} \sin \gamma-\dot{h}_{d}=x_{1} \gamma+x_{1}(\sin \gamma-\gamma)-\dot{h}_{d}
$$

The command referred to flight path angle is chosen as

$$
\gamma_{d}=\frac{-k_{h} \tilde{h}-x_{1}(\sin \gamma-\gamma)+\dot{h}_{d}}{x_{1}},
$$

where the parameter $k_{h}$ donates the control gain.

$\dot{x}_{2}=f_{2}+g_{2} x_{3}+d_{2}$,

$\dot{x}_{3}=f_{3}+g_{3} x_{4}+d_{3}$,

$\dot{x}_{4}=f_{4}+g_{4} u_{4}+d_{4}$,

$u_{2}=\delta_{e}$,

$y_{2}=x_{2}$,

$f_{2}=-\frac{1}{2} \rho x_{1}^{2} S \times 0.6023 \frac{1}{m x_{1}} x_{2}-\frac{\mu-x_{1}^{2} r}{x_{1} r^{2}} \cos x_{2}$

$$
\begin{aligned}
& g_{1}=\frac{1}{2} \rho x_{1}^{2} S \times 0.6023 \frac{1}{m x_{1}}, \\
& f_{2}=0 \\
& g_{2}=1 \\
& f_{3}=\rho x_{1}^{2} S c \frac{\left[C_{M}\left(x_{3}-x_{2}\right)+C_{M}\left(x_{4}\right)-0.0292\left(x_{3}-x_{2}\right)\right]}{2 I_{y y}}
\end{aligned}
$$

$$
g_{3}=\frac{1}{2} \rho x_{1}^{2} S \bar{c} \frac{0.0292}{I_{y y}} .
$$

\section{Controller Design}

And the composited controller, consisting of an adaptive back-stepping method and nonlinear disturbance observer, is designed for a GHV. The NDO is added into the controller for improving performance of the controller.

3.1. New Adaptive Sliding Mode Controller Design. The proposed controller utilizes the back-stepping method while the virtual control inputs can be obtained at each step. The designed adaptive law can compensate for the modeling uncertainties effectively.

3.1.1. Controller for the Velocity Subsystem. The velocity tracking error can be defined as

$$
z_{1}=x_{1}-x_{1 d}
$$

A new sliding mode is chosen as

$$
s_{1}=\dot{z}_{1}+\frac{c_{1} z_{1}}{\left|z_{1}\right|+\delta_{1}^{2} \eta_{1}}+\widehat{d}_{1},
$$

where $\widehat{d}_{1}$ represents the disturbance estimation of $d_{1}$.

The adaptive parameter is chosen as

$$
\dot{\delta}_{1}=-\lambda_{1} \frac{c_{1}\left|z_{1}\right| \delta_{1} \eta_{1}}{\left|z_{1}\right|+\delta_{1}^{2} \eta_{1}} .
$$

When the differentiation of tracking error is taken into the dynamics, the equation can be obtained as

$$
\begin{aligned}
\dot{s}_{1} & =\ddot{z}_{1}+\left[\frac{c_{1} z_{1}}{\left|z_{1}\right|+\delta_{1}^{2} \eta_{1}}\right]+\dot{\hat{d}}_{1} \\
& =\dot{f}_{1}+\left[g_{1} u_{1}\right]^{\prime}+\dot{d}_{1}-\ddot{x}_{1 d}+\left[\frac{c_{1} z_{1}}{\left|z_{1}\right|+\delta_{1}^{2} \eta_{1}}\right]+\dot{\hat{d}}_{1} .
\end{aligned}
$$

The command of throttle setting can be designed as

$$
\begin{aligned}
u_{1}=-g_{1}^{-1} & {\left[\int k_{1,1} s_{1}+k_{1,2} \operatorname{sgn}\left(s_{1}\right) d t\right.} \\
& \left.+f_{1}-\dot{x}_{1 d}+\frac{c_{1} z_{1}}{\left|z_{1}\right|+\delta_{1}^{2} \eta_{1}}+\widehat{d}_{1}\right],
\end{aligned}
$$


where $k_{1,1}$ and $k_{1,2}$ represent the controller parameters, respectively, which determine the convergence rate of this subsystem.

3.1.2. Controller for Altitude Subsystem. The back-stepping method is used to design the controller for altitude subsystem.

Step 1 (the control input design for the flight path angle). The tracking error in this step can be defined as

$$
z_{2}=x_{2}-x_{2 d}
$$

For making the tracking error converge to zero, the sliding mode surface can be designed as

$$
s_{2}=\dot{z}_{2}+\frac{c_{2} z_{2}}{\left|z_{2}\right|+\delta_{2}^{2} \eta_{2}}+\widehat{d}_{2}
$$

where $\hat{d}_{2}$ is the disturbance estimation of $d_{2}$.

The adaptive parameter is chosen as

$$
\dot{\delta}_{2}=-\lambda_{2} \frac{c_{2}\left|z_{2}\right| \delta_{2} \eta_{2}}{\left|z_{2}\right|+\delta_{2}^{2} \eta_{2}} .
$$

If the differentiation is taken into the sliding mode, it can be obtained as

$$
\begin{aligned}
\dot{s}_{2} & =\ddot{z}_{2}+\left[\frac{c_{2} z_{2}}{\left|z_{2}\right|+\delta_{2}^{2} \eta_{2}}\right]+\dot{\vec{d}}_{2} \\
& =\dot{f}_{2}+\left[g_{2} x_{3}\right]^{\prime}+\dot{d}_{2}-\ddot{x}_{2 d}+\left[\frac{c_{2} z_{2}}{\left|z_{2}\right|+\delta_{2}^{2} \eta_{2}}\right]+\dot{\vec{d}}_{2} .
\end{aligned}
$$

It can be obtained as

$$
\begin{array}{r}
x_{3 d}=-g_{2}{ }^{-1}\left[\int k_{2,1} s_{2}+k_{2,2} \operatorname{sgn}\left(s_{2}\right) \mathrm{d} t+f_{2}\right. \\
\left.-\dot{x}_{2 d}+\frac{c_{2} z_{2}}{\left|z_{2}\right|+\delta_{2}^{2} \eta_{2}}+\widehat{d}_{2}\right] .
\end{array}
$$

Step 2 (the control input design for the pitching angle). The tracking error and sliding mode surface can be defined as follows:

$$
\begin{aligned}
& z_{3}=x_{3}-x_{3 d}, \\
& s_{3}=\dot{z}_{3}+\frac{c_{3} z_{3}}{\left|z_{3}\right|+\delta_{3}^{2} \eta_{3}}+\widehat{d}_{3},
\end{aligned}
$$

where $\hat{d}_{3}$ is the disturbance estimation of $d_{3}$.

The adaptive parameter is chosen as

$$
\dot{\delta}_{3}=-\lambda_{3} \frac{c_{3}\left|z_{3}\right| \delta_{3} \eta_{3}}{\left|z_{3}\right|+\delta_{3}^{2} \eta_{3}} .
$$

The equation can be transformed when the derivation of $s_{3}$ is taken into the dynamics

$$
\begin{aligned}
\dot{s}_{3} & =\ddot{z}_{3}+\left[\frac{c_{3} z_{3}}{\left|z_{3}\right|+\delta_{3}^{2} \eta_{3}}\right]+\dot{\hat{d}}_{3} \\
& =\dot{f}_{3}+\left[g_{3} x_{4}\right]^{\prime}+\dot{d}_{3}-\ddot{x}_{3 d}+\left[\frac{c_{3} z_{3}}{\left|z_{3}\right|+\delta_{3}^{2} \eta_{3}}\right]+\dot{\vec{d}}_{3} .
\end{aligned}
$$

It can be obtained as

$$
\begin{array}{r}
x_{4 d}=-g_{3}{ }^{-1}\left[\int k_{3,1} s_{3}+k_{3,2} \operatorname{sgn}\left(s_{3}\right) d t+f_{3}\right. \\
\left.-\dot{x}_{3 d}+\frac{c_{3} z_{3}}{\left|z_{3}\right|+\delta_{3}^{2} \eta_{3}}+\widehat{d}_{3}\right] .
\end{array}
$$

Step 3 (the control input design for the pitching rate angle). The tracking error $z_{4}$ and sliding mode surface $s_{4}$ in this step can be designed as follows:

$$
\begin{aligned}
& z_{4}=x_{4}-x_{4 d}, \\
& s_{4}=\dot{z}_{4}+\frac{c_{4} z_{4}}{\left|z_{4}\right|+\delta_{4}^{2} \eta_{4}}+\widehat{d}_{4},
\end{aligned}
$$

where $\widehat{d}_{4}$ is the disturbance estimation of $d_{4}$.

The adaptive parameter is chosen as

$$
\dot{\delta}_{4}=-\lambda_{4} \frac{c_{4}\left|z_{4}\right| \delta_{4} \eta_{4}}{\left|z_{4}\right|+\delta_{4}^{2} \eta_{4}} .
$$

The equation can be transformed when the derivation of $z_{4}$ is taken into the tracking error.

$$
\begin{aligned}
\dot{s}_{4} & =\ddot{z}_{4}+\left[\frac{c_{4} z_{4}}{\left|z_{4}\right|+\delta_{4}^{2} \eta_{4}}\right]+\dot{\hat{d}}_{4} \\
& =\dot{f}_{4}+\left[g_{4} u_{2}\right]^{\prime}+\dot{d}_{4}-\ddot{x}_{4 d}+\left[\frac{c_{4} z_{4}}{\left|z_{4}\right|+\delta_{4}^{2} \eta_{4}}\right]+\dot{\vec{d}}_{4} .
\end{aligned}
$$

The slide mode controller can be obtained as follows:

$$
\begin{array}{r}
u_{2}=-g_{4}{ }^{-1}\left[\int k_{4,1} s_{4}+k_{4,2} \operatorname{sgn}\left(s_{4}\right) d t+f_{4}\right. \\
\left.-\dot{x}_{4 d}+\frac{c_{4} z_{4}}{\left|z_{4}\right|+\delta_{4}^{2} \eta_{4}}+\widehat{d}_{4}\right] .
\end{array}
$$

3.2. Nonlinear Disturbance Observer Design. Inspired by the works of Zhang et al. [26], Liu et al. [27], and Tian et al. [28], a nonlinear disturbance observer is designed as follows.

3.2.1. NDO for Velocity Subsystem. A nonlinear disturbance observer for (1) is designed as 


$$
\begin{gathered}
{\left[\begin{array}{c}
\dot{\hat{x}}_{1} \\
\dot{\hat{d}}_{1}
\end{array}\right]=\left[\begin{array}{cc}
A_{12} & 1 \\
0 & 0
\end{array}\right]\left[\begin{array}{l}
\hat{x}_{1} \\
\hat{d}_{1}
\end{array}\right]+\left[\begin{array}{c}
A_{11} \\
0
\end{array}\right]\left(x_{3}-x_{2}\right)} \\
+\left[\begin{array}{c}
B_{1} \\
0
\end{array}\right] u_{1}-\left[\begin{array}{l}
L_{11} \\
L_{12}
\end{array}\right]\left(\hat{x}_{1}-x_{1}\right), \\
A_{11}=-0.0043378 \frac{\rho x_{1}^{2} S}{2 m}, A_{12}=0, \\
B_{1}=0.00336 \beta_{c} \frac{\rho x_{1}^{2} S}{2 m},
\end{gathered}
$$

where $L_{11}=A_{11}+2 \Lambda_{1}, L_{12}=\Lambda_{1}^{2}$, and $\Lambda_{1}>0$.

3.2.2. NDO for Altitude Subsystem. Similarly, an NDO for (3) is obtained as

$$
\left[\begin{array}{c}
\dot{\hat{h}} \\
\dot{\hat{d}}_{h}
\end{array}\right]=\left[\begin{array}{cc}
H_{11} & 1 \\
0 & 0
\end{array}\right]\left[\begin{array}{c}
\widehat{h} \\
\widehat{d}_{h}
\end{array}\right]-\left[\begin{array}{c}
H_{21} \\
H_{22}
\end{array}\right](\widehat{h}-h), \quad H_{11}=x_{c},
$$

where $H_{21}=H_{11}+2 \Lambda_{H}, H_{22}=\Lambda_{H}^{2}$, and $\Lambda_{H}>0$.

A nonlinear disturbance observer for (2) is designed as

$$
\begin{gathered}
{\left[\begin{array}{c}
\dot{\hat{x}}_{2} \\
\dot{\hat{d}}_{2}
\end{array}\right]=\left[\begin{array}{cc}
A_{22} & 1 \\
0 & 0
\end{array}\right]\left[\begin{array}{l}
\widehat{x}_{2} \\
\widehat{d}_{2}
\end{array}\right]+\left[\begin{array}{c}
A_{21} \\
0
\end{array}\right] x_{3}-\left[\begin{array}{c}
L_{21} \\
L_{22}
\end{array}\right]\left(\widehat{x}_{2}-x 2\right),} \\
A_{21}=-0.6427 \frac{\rho x_{1}^{2} S}{2 m x_{1}}, A_{22}=0.6427 \frac{\rho x_{1}^{2} S}{2 m x_{1}}
\end{gathered}
$$

where $L_{21}=A_{21}+2 \Lambda_{2}, L_{22}=\Lambda_{2}^{2}$, and $\Lambda_{2}>0$.

A nonlinear disturbance observer for (4) is designed as

$$
\begin{array}{r}
{\left[\begin{array}{c}
\dot{\hat{x}}_{3} \\
\dot{\hat{d}}_{3}
\end{array}\right]=\left[\begin{array}{cc}
A_{31} & 1 \\
0 & 0
\end{array}\right]\left[\begin{array}{l}
\hat{x}_{3} \\
\hat{d}_{3}
\end{array}\right]+\left[\begin{array}{c}
A_{32} \\
0
\end{array}\right] x_{4}-\left[\begin{array}{c}
L_{31} \\
L_{32}
\end{array}\right]\left(\hat{x}_{3}-x_{3}\right),} \\
A_{31}=0, A_{32}=1,
\end{array}
$$

where $L_{31}=A_{31}+2 \Lambda_{3}, L_{32}=\Lambda_{3}^{2}$, and $\Lambda_{3}>0$.

Similarly, a nonlinear disturbance observer for (5) is designed as

$$
\begin{aligned}
{\left[\begin{array}{c}
\dot{\hat{x}}_{4} \\
\dot{\hat{d}}_{4}
\end{array}\right]=} & {\left[\begin{array}{cc}
A_{42} & 1 \\
0 & 0
\end{array}\right]\left[\begin{array}{l}
\hat{x}_{4} \\
\hat{d}_{4}
\end{array}\right]+\left[\begin{array}{c}
A_{41} \\
0
\end{array}\right] x_{3}+\left[\begin{array}{c}
B_{2} \\
0
\end{array}\right] u_{2} } \\
& -\left[\begin{array}{c}
L_{41} \\
L_{42}
\end{array}\right]\left(\hat{x}_{4}-x_{4}\right), \\
A_{41}= & \frac{\rho x_{1}^{2} S \bar{c}}{2 I_{y y}}\left(0.036617-c_{e}\right), A_{42}=-0.2289 \frac{\rho x_{1} S \bar{c}^{2}}{4 I_{y y}}, \\
B_{2}= & \frac{c_{e} \rho x_{1}^{2} S \bar{c}}{2 I_{y y}},
\end{aligned}
$$

where $L_{41}=A_{41}+2 \Lambda_{4}, L_{42}=\Lambda_{4}^{2}$, and $\Lambda_{4}>0$.

\section{Stability Analysis}

4.1. Convergence of SMC. The stability of the closed control system is proved by the Lyapunov stability theory.

Firstly, a Lyapunov function is chosen as

$$
F=F_{1}+F_{2}+F_{3}+F_{4}=\frac{1}{2} s_{1}^{2}+\frac{1}{2} s_{2}^{2}+\frac{1}{2} s_{3}^{2}+\frac{1}{2} s_{4}^{2} .
$$

The derivation of the Lyapunov function is obtained as

$$
\dot{F}=\dot{F}_{1}+\dot{F}_{2}+\dot{F}_{3}+\dot{F}_{4}=s_{1} \dot{s}_{1}+s_{2} \dot{s}_{2}+s_{3} \dot{s}_{3}+s_{4} \dot{s}_{4} .
$$

Step 1 (stability analysis for the velocity subsystem). The derivation of the Lyapunov function for system (11) can be obtained as

$$
\begin{aligned}
\dot{F}_{1}= & \left\{-k_{1,1} s_{1}-k_{1,2} \operatorname{sgn}\left(s_{1}\right)-\dot{f}_{1}+\ddot{x}_{1 d}-\left[\frac{c_{1} z_{1}}{\left|z_{1}\right|+\delta_{1}^{2} \eta_{1}}\right]^{\prime}-\widehat{d}_{1}\right. \\
& \left.+\dot{f}_{1}+\dot{d}_{1}-\ddot{x}_{1 d}+\left[\frac{c_{1} z_{1}}{\left|z_{1}\right|+\delta_{1}^{2} \eta_{1}}\right]^{\prime}+\widehat{d}_{1}\right\} s_{1} \\
= & -k_{1,1} s_{1}^{2}-k_{1,2}\left|s_{1}\right|+\dot{d}_{1} s_{1} .
\end{aligned}
$$

If the parameters of the controller (26) is chosen to meet $k_{1,2} \geq D_{1,2} \geq\left|\dot{d}_{1}\right|$, it can be obtained as

$$
\dot{F}_{1} \leq-k_{1,1} s_{1}^{2} \leq 0 .
$$

Step 2 (stability analysis for the angles)

(a) Flight path angle:

$$
\begin{aligned}
\dot{F}_{2}=s_{2} \dot{s}_{2}= & \left\{-k_{2,1} s_{2}-k_{2,2} \operatorname{sgn}\left(s_{2}\right)-\dot{f}_{2}+\ddot{x}_{2 d}-\left[\frac{c_{2} z_{2}}{\left|z_{2}\right|+\delta_{2}^{2} \eta_{2}}\right]^{\prime}\right. \\
& \left.-\widehat{d}_{2}+\dot{f}_{2}+\dot{d}_{2}-\ddot{x}_{2 d}+\left[\frac{c_{2} z_{2}}{\left|z_{2}\right|+\delta_{2}^{2} \eta_{2}}\right]^{\prime}+\widehat{d}_{2}\right\} s_{2} \\
= & -k_{2,1} s_{2}^{2}-k_{2,2}\left|s_{2}\right|+\dot{d}_{2} s_{2} .
\end{aligned}
$$


If $k_{2,2} \geq D_{2,2} \geq\left|\dot{d}_{2}\right|$ is satisfied, it can be obtained as

$$
\dot{F}_{2} \leq-k_{2,1} s_{2}^{2} \leq 0
$$

(b) Pitching angle:

$$
\begin{aligned}
\dot{F}_{3}= & \left\{-k_{3,1} s_{3}-k_{3,2} \operatorname{sgn}\left(s_{3}\right)-\dot{f}_{3}+\ddot{x}_{3 d}-\left[\frac{c_{3} z_{3}}{\left|z_{3}\right|+\delta_{3}^{2} \eta_{3}}\right]^{\prime}-\widehat{d}_{3}\right. \\
& \left.+\dot{f}_{3}+\dot{d}_{3}-\ddot{x}_{3 d}+\left[\frac{c_{3} z_{3}}{\left|z_{3}\right|+\delta_{3}^{2} \eta_{3}}\right]^{\prime}+\widehat{d}_{3}\right\} s_{3} \\
= & -k_{3,1} s_{3}^{2}-k_{3,2}\left|s_{3}\right|+\dot{d}_{3} s_{3} .
\end{aligned}
$$

If $k_{3,2} \geq D_{3,2} \geq\left|\dot{d}_{3}\right|$ is satisfied, it can be obtained as

$$
\dot{F}_{3} \leq-k_{3,1} s_{3}^{2} \leq 0
$$

(c) Pitching rate:

$$
\begin{aligned}
\dot{F}_{4}=\{- & k_{4,1} s_{4}-k_{4,2} \operatorname{sgn}\left(s_{4}\right)-\dot{f}_{4}+\ddot{x}_{4 d}-\left[\frac{c_{4} z_{4}}{\left|z_{4}\right|+\delta_{4}^{2} \eta_{4}}\right]^{\prime}-\widehat{d}_{4} \\
& \left.+\dot{f}_{4}+\dot{d}_{4}-\ddot{x}_{4 d}+\left[\frac{c_{4} z_{4}}{\left|z_{4}\right|+\delta_{4}^{2} \eta_{4}}\right]^{\prime}+\widehat{d}_{4}\right\} s_{4} \\
= & -k_{4,1} s_{4}^{2}-k_{4,2}\left|s_{4}\right|+\dot{d}_{4} s_{4} .
\end{aligned}
$$

If $k_{4,2} \geq D_{4,2} \geq\left|\dot{d}_{4}\right|$ is satisfied, it can be obtained as

$$
\dot{F} \leq-k_{4,1} s_{4}^{2} \leq 0 .
$$

The Lyapunov stability is proved.

$$
\begin{aligned}
F & =F_{1}+F_{2}+F_{3}+F_{4}=\frac{1}{2} s_{1}^{2}+\frac{1}{2} s_{2}^{2}+\frac{1}{2} s_{3}^{2}+\frac{1}{2} s_{4}^{2} \geq 0, \\
\dot{F} & =\dot{F}_{1}+\dot{F}_{2}+\dot{F}_{3}+\dot{F}_{4}=s_{1} \dot{s}_{1}+s_{2} \dot{s}_{2}+s_{3} \dot{s}_{3}+s_{4} \dot{s}_{4} \\
& \leq-k_{1,1} s_{1}^{2}-k_{2,1} s_{2}^{2}-k_{3,1} s_{3}^{2}-k_{4,1} s_{4}^{2} .
\end{aligned}
$$

4.2. Convergence of Tracking Error. The Lyapunov function is chosen as

$$
\begin{aligned}
W= & \frac{1}{2} z_{1}^{2}+\frac{1}{2 \lambda_{1}} \delta_{1}^{2}+\frac{1}{2} \tilde{h}^{2}+\frac{1}{2} z_{3}^{2}+\frac{1}{2 \lambda_{2}} \delta_{2}^{2} \\
& +\frac{1}{2} z_{4}^{2}+\frac{1}{2 \lambda_{3}} \delta_{3}^{2}+\frac{1}{2} z_{5}^{2}+\frac{1}{2 \lambda_{4}} \delta_{4}^{2},
\end{aligned}
$$

where $z_{1}, \tilde{h}, z_{3}, z_{4}$, and $z_{5}$ donate the tracking error, respectively.

The derivation of the Lyapunov function can be obtained as

$$
\begin{array}{r}
\dot{W}=z_{1} \dot{z}_{1}+\frac{1}{\lambda_{1}} \delta_{1} \dot{\delta}_{1}+\tilde{h} \dot{\tilde{h}}+z_{3} \dot{z}_{3}+\frac{1}{\lambda_{2}} \delta_{2} \dot{\delta}_{2} \\
+z_{4} \dot{z}_{4}+\frac{1}{2 \lambda_{3}} \delta_{3} \dot{\delta}_{3}+z_{5} \dot{z}_{5}+\frac{1}{2 \lambda_{4}} \delta_{4} \dot{\delta}_{4}
\end{array}
$$

Step 1 (stability analysis for velocity). The Lyapunov function for system (11) is chosen as

$$
W_{1}=\frac{1}{2} z_{1}^{2}+\frac{1}{2 \lambda_{1}} \delta_{1}^{2}
$$

The derivation of the Lyapunov function can be obtained as

$$
\begin{aligned}
\dot{W}_{1} & =z_{1} \dot{z}_{1}+\frac{1}{\lambda_{1}} \delta_{1} \dot{\delta}_{1}=-\frac{c_{1} z_{1}^{2}}{\left|z_{1}\right|+\delta_{1}^{2} \eta_{1}}-\widehat{d}_{1} z_{1}+\frac{1}{\lambda_{1}} \delta_{1} \dot{\delta}_{1} \\
& =-c_{1}\left|z_{1}\right|+\frac{c_{1}\left|z_{1}\right| \delta_{1}^{2} \eta_{1}}{\left|z_{1}\right|+\delta_{1}^{2} \eta_{1}}-\widehat{d}_{1} z_{1}-\frac{c_{1}\left|z_{1}\right| \delta_{1}^{2} \eta_{1}}{\left|z_{1}\right|+\delta_{1}^{2} \eta_{1}} \\
& =-c_{1}\left|z_{1}\right|-\widehat{d}_{1} z_{1} .
\end{aligned}
$$

The $c_{1}$ is chosen to satisfy the inequation

$$
c_{1} \geq D_{1,1} \geq\left|\widehat{d}_{1}\right|
$$

It can be obtained as

$$
\dot{W}_{1}=-c_{1}\left|z_{1}\right|-\widehat{d}_{1} z_{1} \leq-\sigma_{1}\left|z_{1}\right|
$$

where $\sigma_{1}=c_{1}-D_{1,1}$ is a positive constant and $\dot{W}_{1} \leq 0$ is satisfied.

Step 2 (stability analysis for the altitude subsystem).

(a) Altitude:

$$
W_{h}=\frac{1}{2} \tilde{h}^{2}
$$

The derivation of $W_{h}$ can be obtained as

$$
\begin{aligned}
\dot{W}_{h}=\tilde{h}\left[x_{1} \frac{-k_{h} \tilde{h}-x_{1}\left(\sin x_{2}-x_{2}\right)+\dot{h}_{d}}{x_{1}} 1\right. \\
\left.\quad+x_{1}\left(\sin x_{2}-x_{2}\right)-\dot{h}_{d}\right] \\
=-k_{h} \tilde{h}^{2}
\end{aligned}
$$

where $k_{h}$ is a positive constant and $\dot{W}_{h} \leq 0$ is satisfied.

(b) Flight path angle: the Lyapunov function is chosen as

$$
W_{2}=\frac{1}{2} z_{2}^{2}+\frac{1}{2 \lambda_{2}} \delta_{2}^{2} \text {. }
$$

The derivation of $W_{2}$ can be obtained as 


$$
\begin{aligned}
\dot{W}_{2} & =z_{2} \dot{z}_{2}+\frac{1}{\lambda_{2}} \delta_{2} \dot{\delta}_{2}=-\frac{c_{2} z_{2}^{2}}{\left|z_{2}\right|+\delta_{2}^{2} \eta_{2}}-\widehat{d}_{2} z_{2}+\frac{1}{\lambda_{2}} \delta_{2} \dot{\delta}_{2} \\
& =-c_{2}\left|z_{2}\right|+\frac{c_{2}\left|z_{2}\right| \delta_{2}^{2} \eta_{2}}{\left|z_{2}\right|+\delta_{2}^{2} \eta_{2}}-\widehat{d}_{2} z_{2}-\frac{c_{2}\left|z_{2}\right| \delta_{2}^{2} \eta_{2}}{\left|z_{2}\right|+\delta_{2}^{2} \eta_{2}} \\
& =-c_{2}\left|z_{2}\right|-\widehat{d}_{2} z_{2} .
\end{aligned}
$$

The parameter $c_{2}$ is chosen to satisfy

$$
\begin{gathered}
c_{2} \geq D_{2,1} \geq\left|\hat{d}_{2}\right|, \\
\dot{W}_{2} \leq-\sigma_{2}\left|z_{2}\right|,
\end{gathered}
$$

where $\sigma_{2}=c_{2}-D_{2,1}$ is a positive constant and $\dot{W}_{2} \leq 0$ is satisfied.

(c) Pitching angle: the Lyapunov function is chosen as

$$
W_{3}=\frac{1}{2} z_{3}^{2}+\frac{1}{2 \lambda_{3}} \delta_{3}^{2} .
$$

The derivation of $W_{3}$ can be obtained as

$$
\begin{aligned}
\dot{W}_{3} & =z_{3} \dot{z}_{3}+\frac{1}{\lambda_{3}} \delta_{3} \dot{\delta}_{3}=-\frac{c_{3} z_{3}^{2}}{\left|z_{3}\right|+\delta_{3}^{2} \eta_{3}}-\widehat{d}_{3} z_{3}+\frac{1}{\lambda_{3}} \delta_{3} \dot{\delta}_{3} \\
& =-c_{3}\left|z_{3}\right|+\frac{c_{3}\left|z_{3}\right| \delta_{3}^{2} \eta_{3}}{\left|z_{3}\right|+\delta_{3}^{2} \eta_{3}}-\widehat{d}_{3} z_{3}-\frac{c_{3}\left|z_{3}\right| \delta_{3}^{2} \eta_{3}}{\left|z_{3}\right|+\delta_{3}^{2} \eta_{3}} \\
& =-c_{3}\left|z_{3}\right|-\widehat{d}_{3} z_{3} .
\end{aligned}
$$

If the parameters $c_{3}$ and $\widehat{d}_{3}$ satisfy $c_{3} \geq D_{3,1} \geq\left|\widehat{d}_{3}\right|$, it can be obtained as

$$
\dot{W}_{3}=-c_{3}\left|z_{3}\right|-\widehat{d}_{3} z_{3} \leq-\sigma_{3}\left|z_{3}\right|,
$$

where $\sigma_{3}=c_{3}-D_{3,1}$ is a positive constant.

(d) Pitching rate: the Lyapunov function is chosen as

$$
W_{4}=\frac{1}{2} z_{4}^{2}+\frac{1}{2 \lambda_{4}} \delta_{4}^{2} .
$$

The derivation of $W_{4}$ can be obtained as

$$
\begin{aligned}
\dot{W}_{4} & =z_{4} \dot{z}_{4}+\frac{1}{\lambda_{4}} \delta_{4} \dot{\delta}_{4}=-\frac{c_{4} z_{4}^{2}}{\left|z_{4}\right|+\delta_{4}^{2} \eta_{4}}-\widehat{d}_{4} z_{4}+\frac{1}{\lambda_{4}} \delta_{4} \dot{\delta}_{4} \\
& =-c_{4}\left|z_{4}\right|+\frac{c_{4}\left|z_{4}\right| \delta_{4}^{2} \eta_{4}}{\left|z_{4}\right|+\delta_{4}^{2} \eta_{4}}-\widehat{d}_{4} z_{4}-\frac{c_{4}\left|z_{4}\right| \delta_{4}^{2} \eta_{4}}{\left|z_{4}\right|+\delta_{4}^{2} \eta_{4}} \\
& =-c_{4}\left|z_{4}\right|-\widehat{d}_{4} z_{4} .
\end{aligned}
$$

If the $c_{4}$ is chosen to satisfy $c_{4} \geq D_{4,1} \geq\left|\widehat{d}_{4}\right|$, it can be obtained as

$$
\dot{W}_{4} \leq-\sigma_{4}\left|z_{4}\right|,
$$

where $\sigma_{4}=c_{4}-D_{4,1}$ is a positive constant. Thus, $\dot{W}_{4} \leq 0$ is satisfied.

$$
\begin{aligned}
W= & \frac{1}{2} z_{1}^{2}+\frac{1}{2 \lambda_{1}} \delta_{1}^{2}+\frac{1}{2} z_{2}^{2}+\frac{1}{2} z_{3}^{2}+\frac{1}{2 \lambda_{2}} \delta_{2}^{2} \\
& +\frac{1}{2} z_{4}^{2}+\frac{1}{2 \lambda_{3}} \delta_{3}^{2}+\frac{1}{2} z_{5}^{2}+\frac{1}{2 \lambda_{4}} \delta_{4}^{2}, \\
\dot{W}= & z_{1} \dot{z}_{1}+\frac{1}{\lambda_{1}} \delta_{1} \dot{\delta}_{1}+z_{2} \dot{z}_{2}+z_{3} \dot{z}_{3}+\frac{1}{\lambda_{2}} \delta_{2} \dot{\delta}_{2} \\
& +z_{4} \dot{z}_{4}+\frac{1}{2 \lambda_{3}} \delta_{3} \dot{\delta} 3+z_{5} \dot{z}_{5}+\frac{1}{2 \lambda_{4}} \delta_{4} \dot{\delta}_{4}, \\
\leq & -\sigma_{1}\left|z_{1}\right|-k_{h} \tilde{h}^{2}-\sigma_{2}\left|z_{2}\right|-\sigma_{3}\left|z_{3}\right|-\sigma_{4}\left|z_{4}\right| .
\end{aligned}
$$

Then it can be obtained as

$$
\begin{aligned}
& W \geq 0, \\
& \dot{W} \leq 0 .
\end{aligned}
$$

The convergence of tracking error is proved now.

\section{Simulation}

In this section, the effectiveness and performance of the developed controller are verified by simulations. The longitudinal model is considered under its cruise flight condition. The initial values are chosen as $V=15060 \mathrm{ft} / \mathrm{s}, h=110000 \mathrm{ft}$, $\gamma=0 \mathrm{rad}, q=0 \mathrm{rad} / \mathrm{s}$, and $\theta=0.01 \mathrm{rad}$, respectively.

The controller parameters are chosen as

$$
\begin{aligned}
k_{h} & =2, \\
\lambda_{1} & =0.005, \\
c_{1} & =15.7, \\
\eta_{1} & =0.1, \\
\lambda_{2} & =0.3, \\
c_{2} & =0.5, \\
\eta_{2} & =0.007, \\
\lambda_{3} & =0.1, \\
c_{3} & =11.2, \\
\eta_{3} & =0.01, \\
\lambda_{4} & =0.05, \\
c_{4} & =8.7, \\
\eta_{4} & =0.01, \\
\Lambda_{1} & =2.6, \\
\Lambda_{H} & =2.2, \\
\Lambda_{2} & =3.1, \\
\Lambda_{3} & =3.1, \\
\Lambda_{4} & =3.3 .
\end{aligned}
$$

The external disturbances are chosen to be $5 \times 10^{-4}$ $\cos (0.5 t), 5 \times 10^{-4} \cos (0.5 t), 1 \times 10^{-2} \cos (0.5 t)$, and $5 \times$ $10^{-3} \cos (0.5 t)$ for the system (11) and (16).

In this part, the square wave and step are applied in command generator, respectively. 


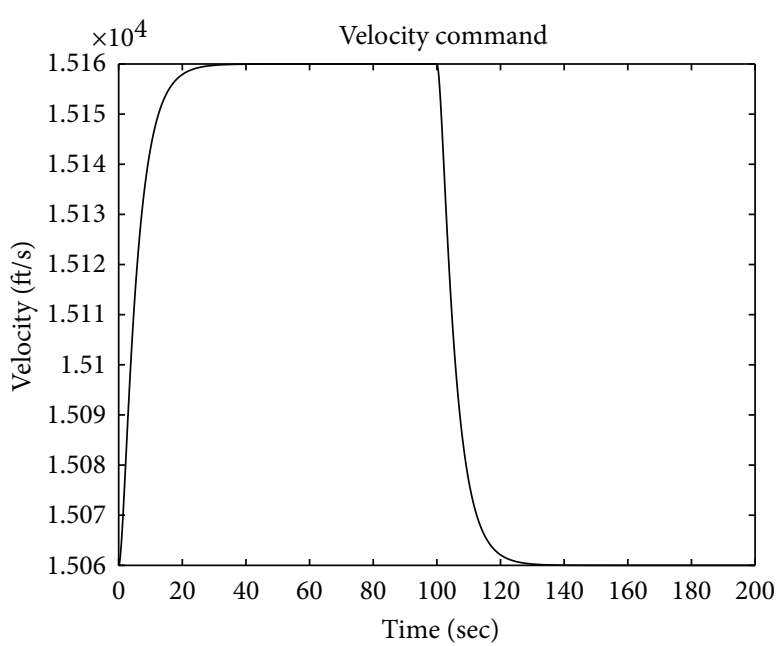

(a)

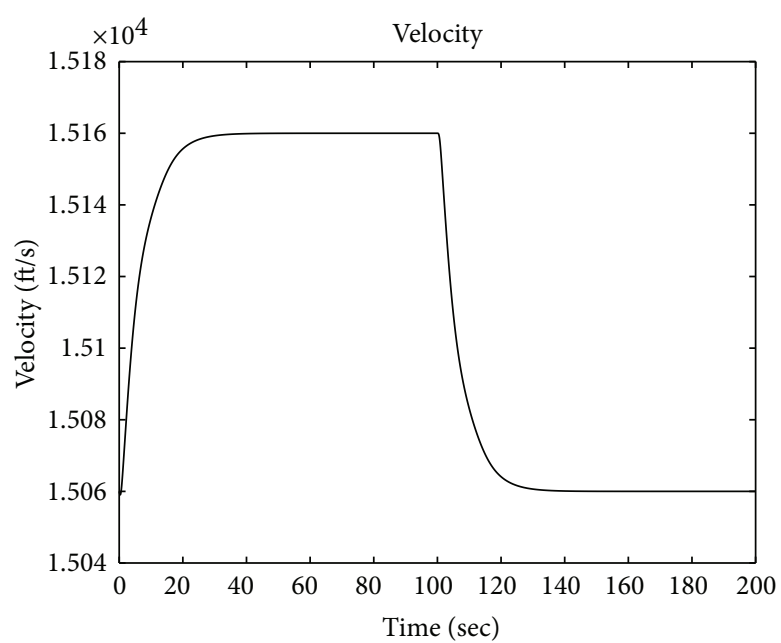

(c)

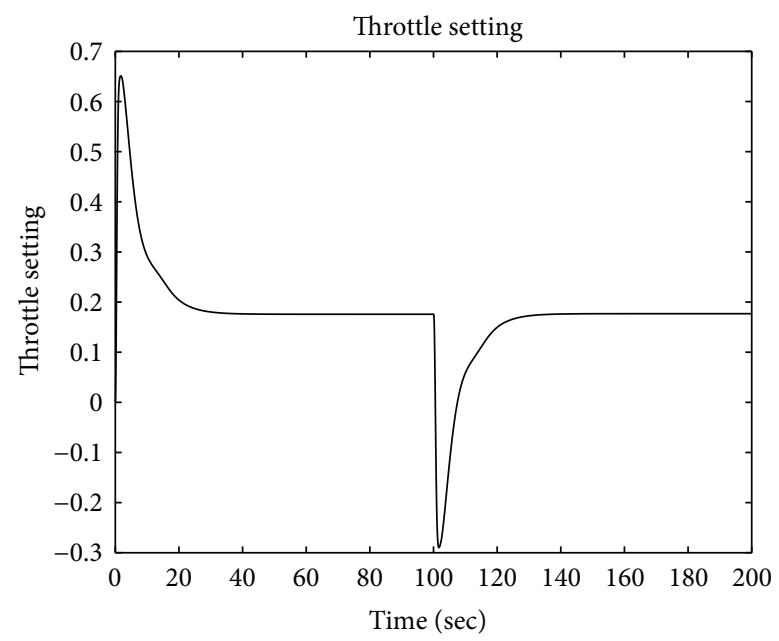

(e)

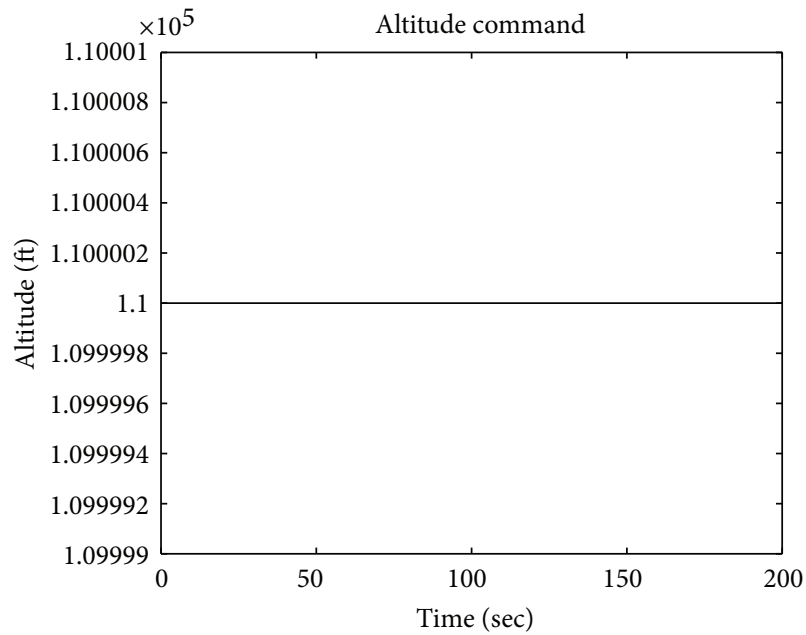

(b)

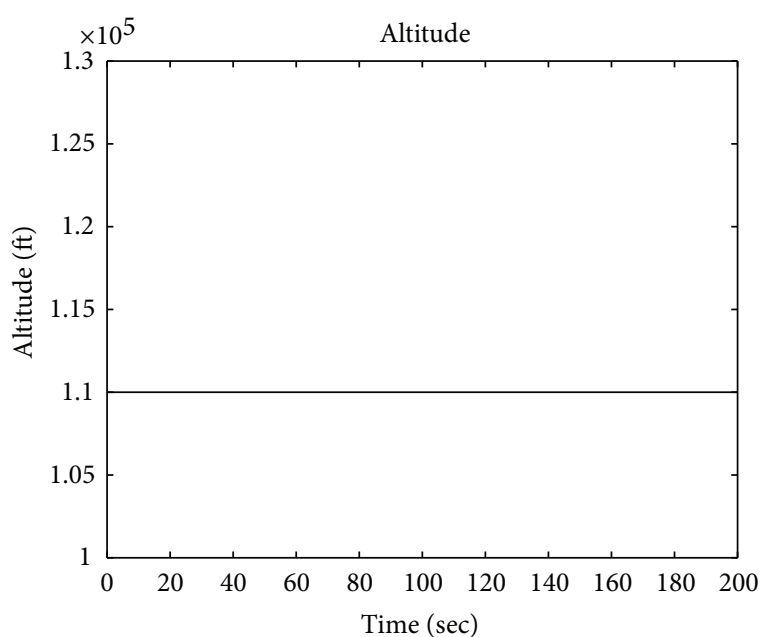

(d)

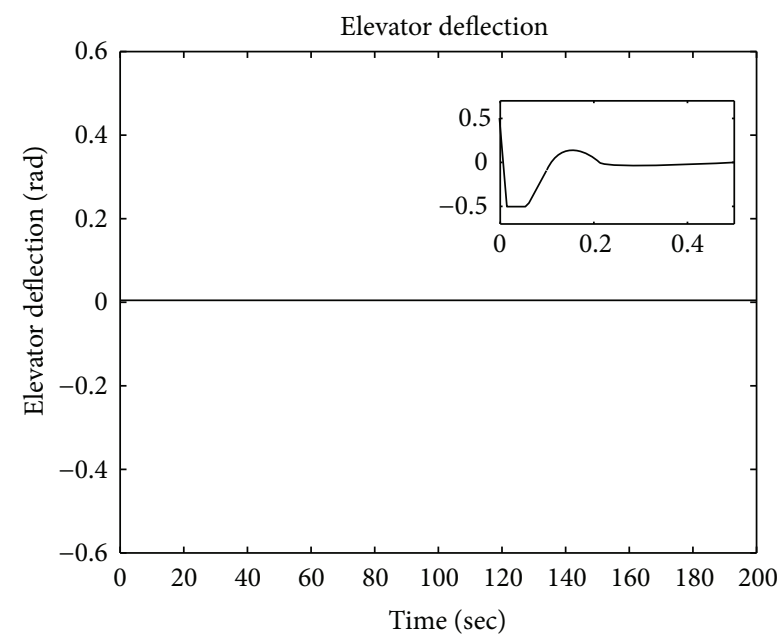

(f)

FIGURE 1: Velocity command, response, and controller. The uncertainty terms are added into the system. 

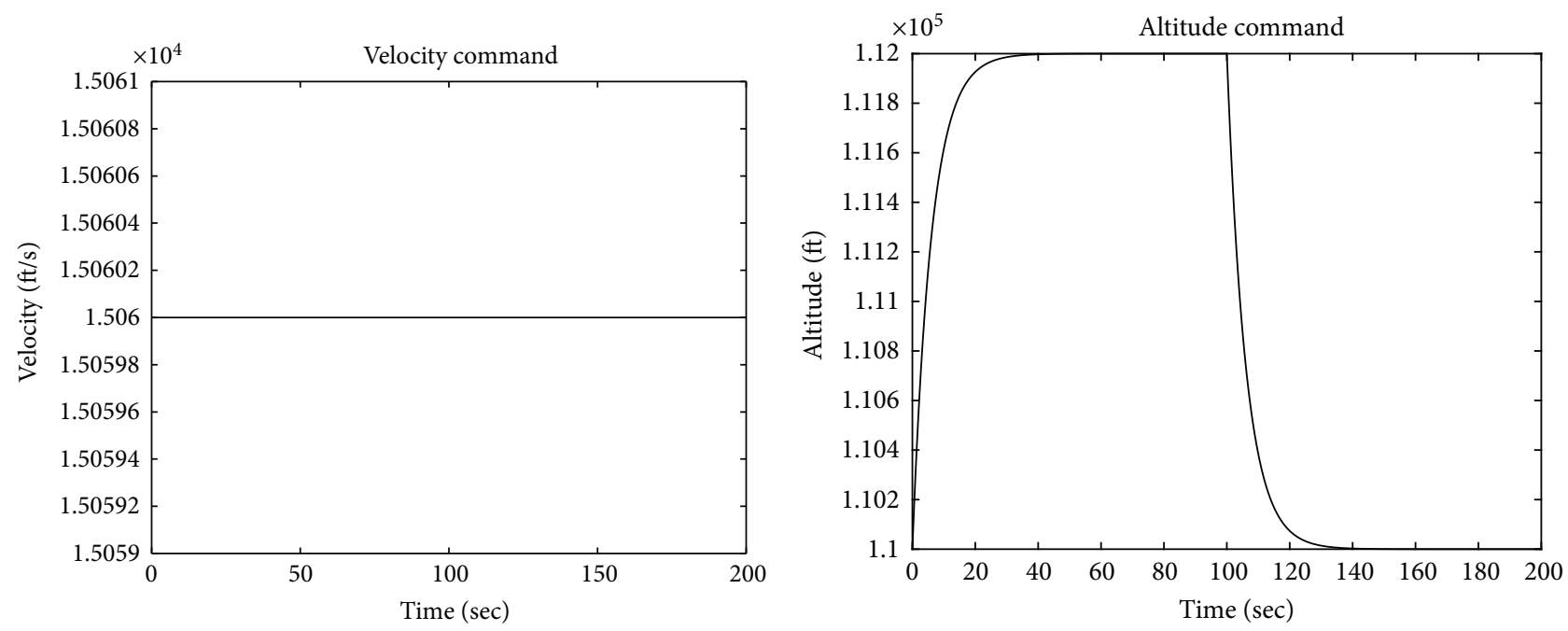

(a)
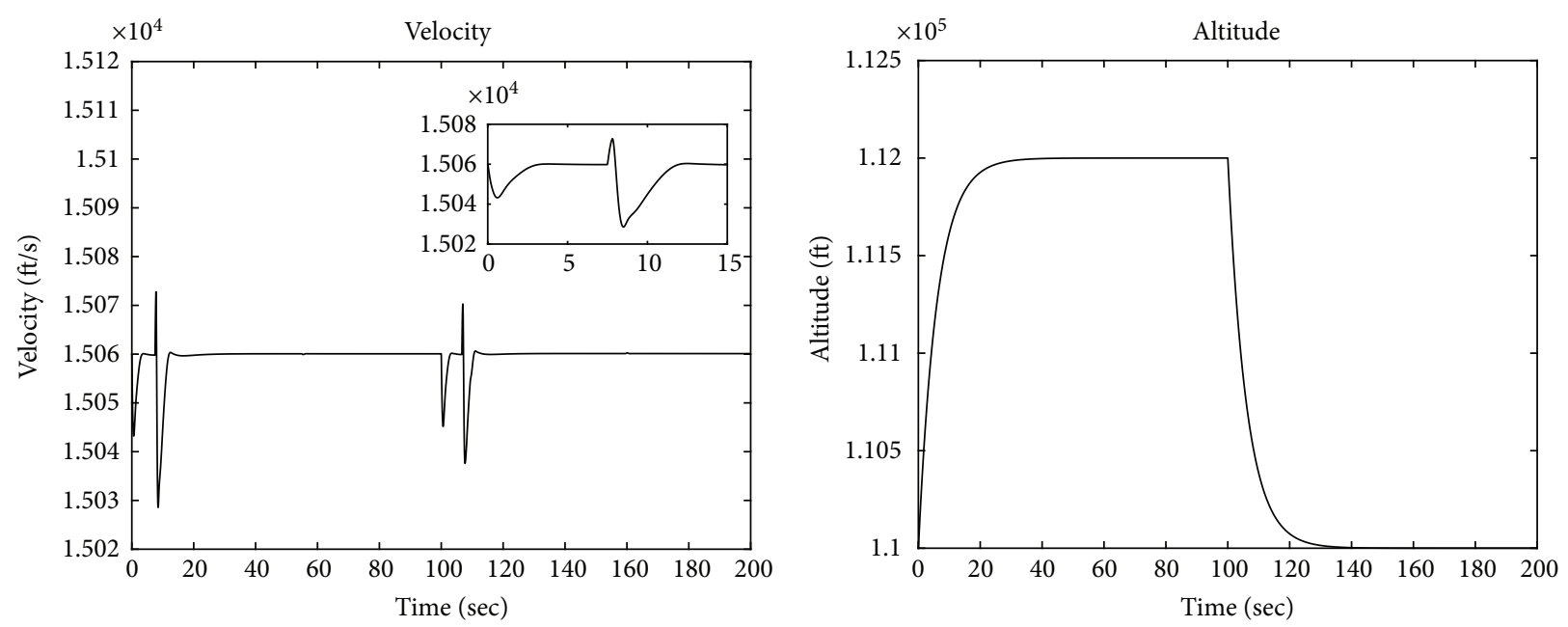

(c)

(d)

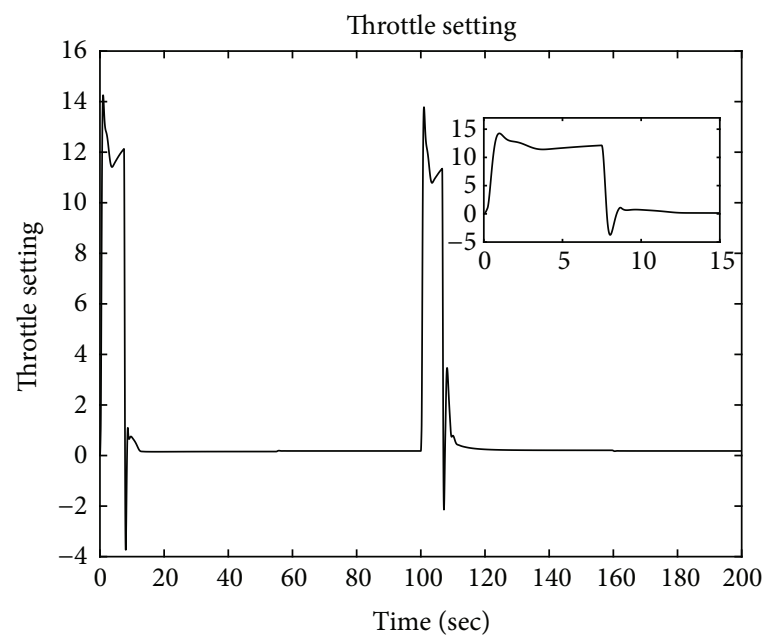

(e)

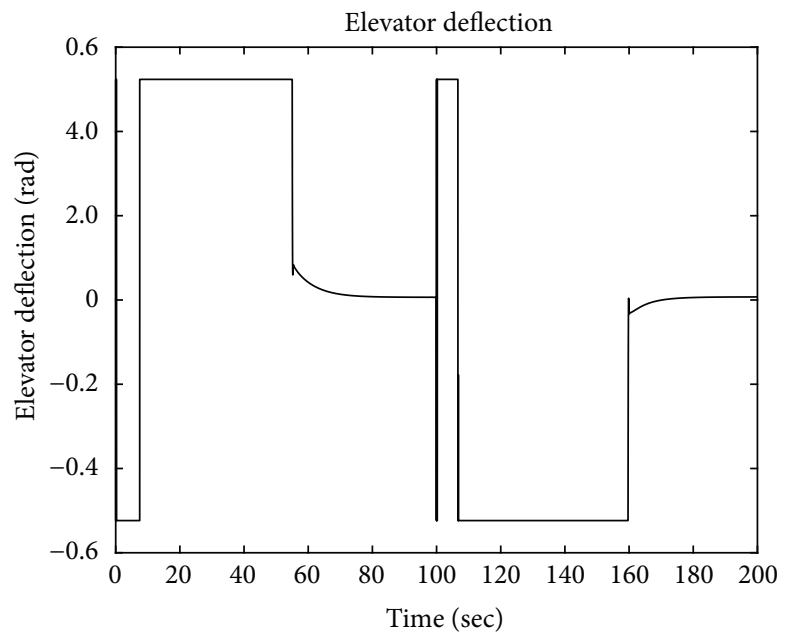

(f)

FIgURE 2: Altitude command, response, and controller. The uncertainty terms are added into the system. 


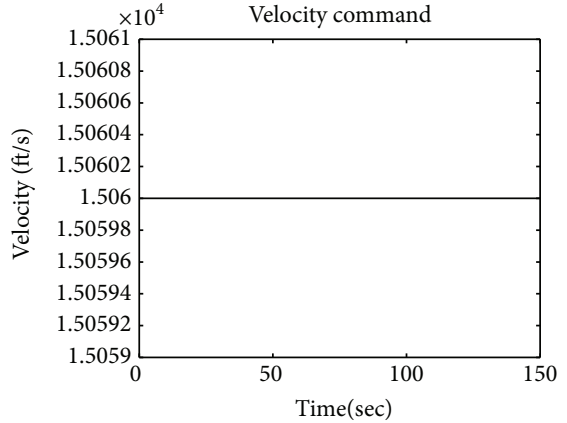

(a)

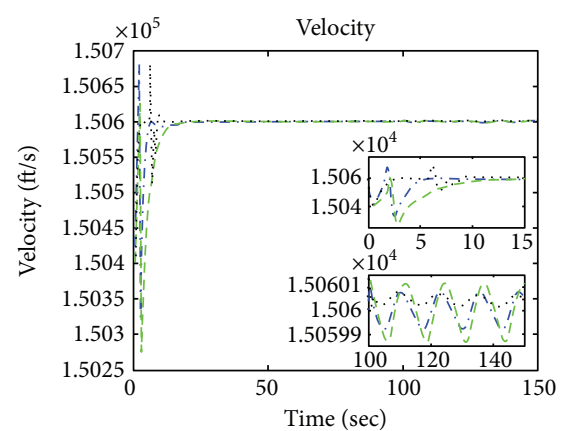

- - - Back-stepping

-. - Back-stepping with DOB

Adaptive back-stepping

(c)

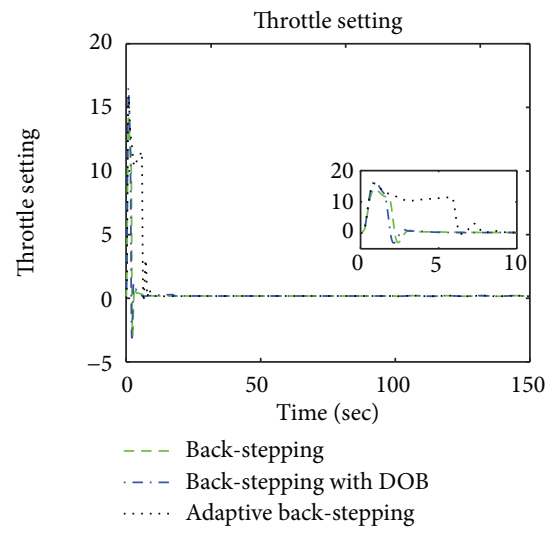

(e)

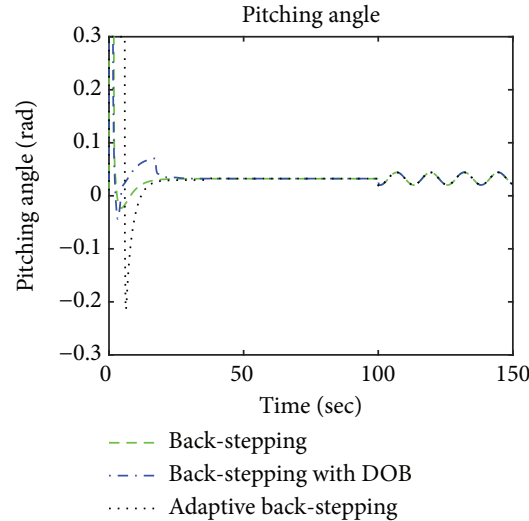

(h)

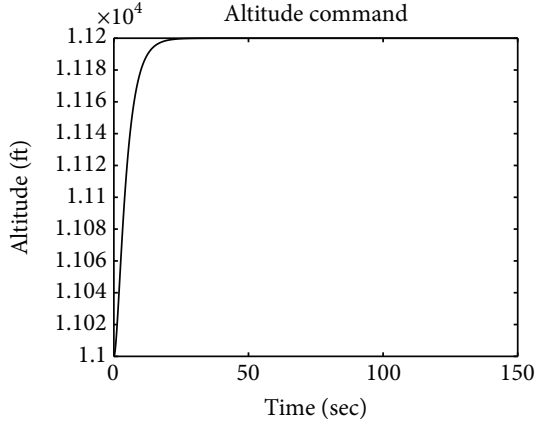

(b)

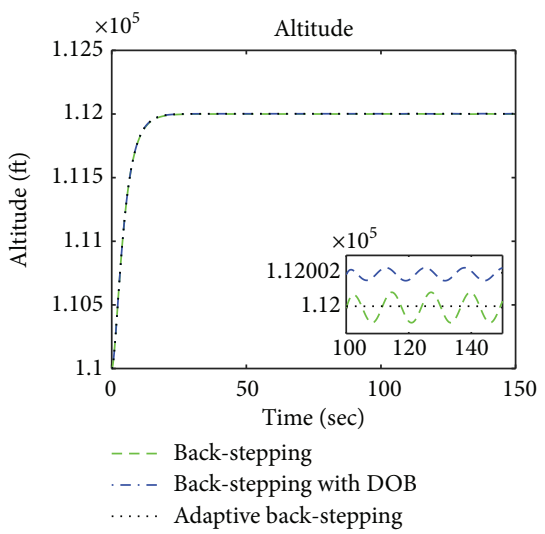

(d)

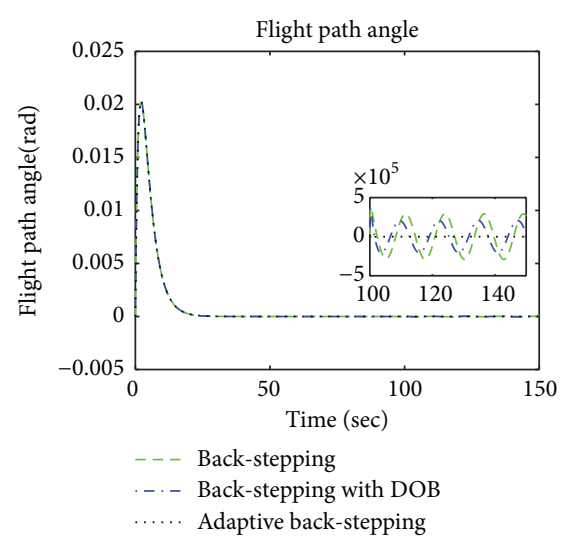

(g)
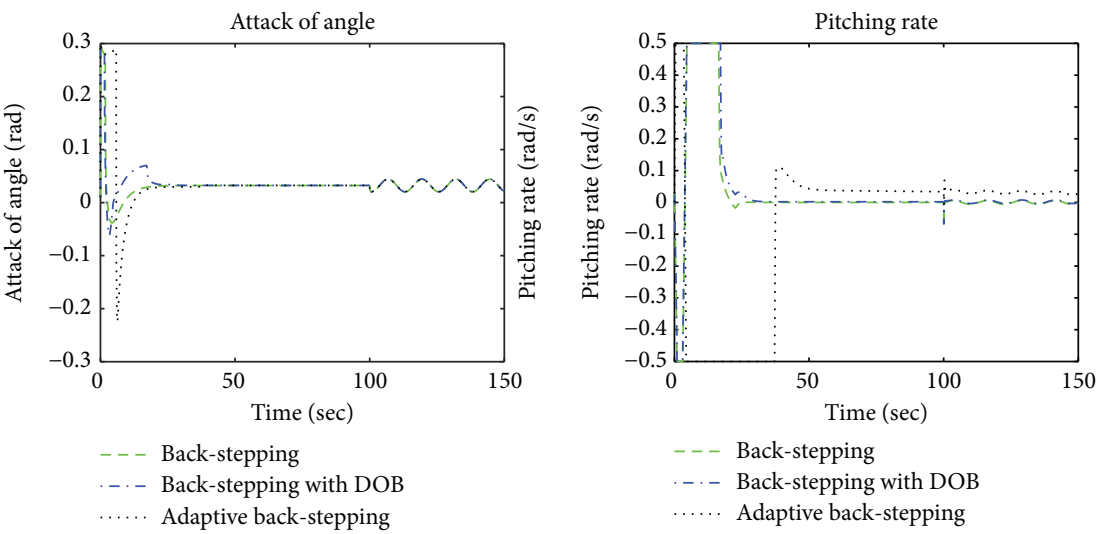

- - Back-stepping

. -. - Back-stepping with DOB

..... Adaptive back-stepping

(i) (j)

Figure 3: Altitude command, response, and controller. The uncertainty terms and external disturbances are added into the system. 

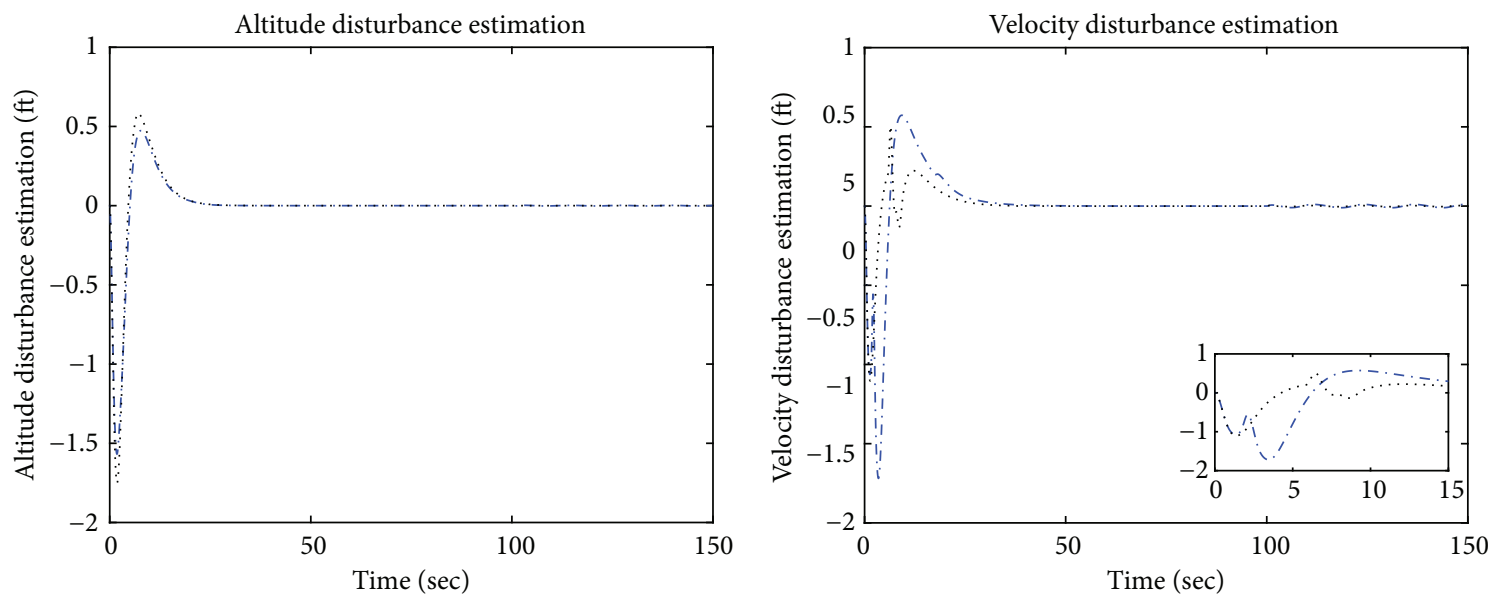

Adaptive back-stepping

...- Back-stepping with DOB

- - Back-stepping with DOB

..... Adaptive back-stepping

(a)

(b)
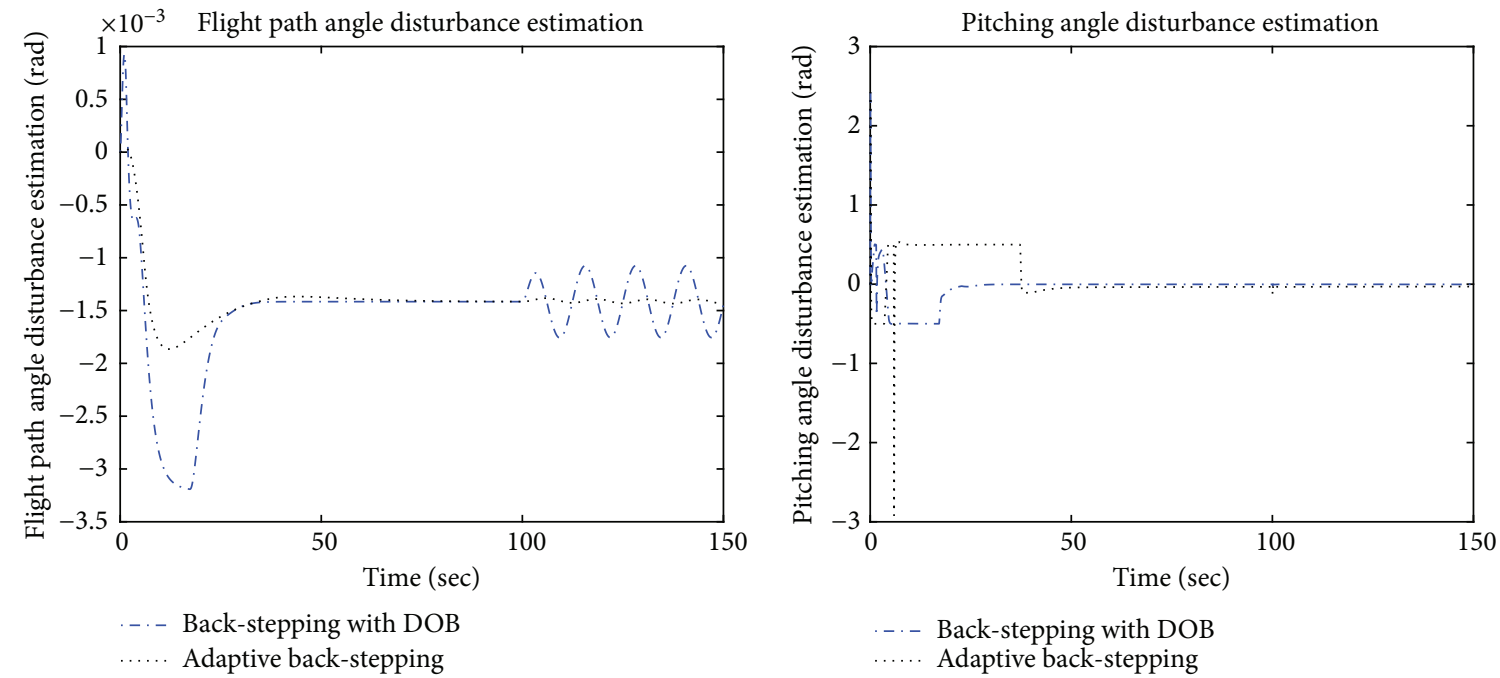

(c)

(d)

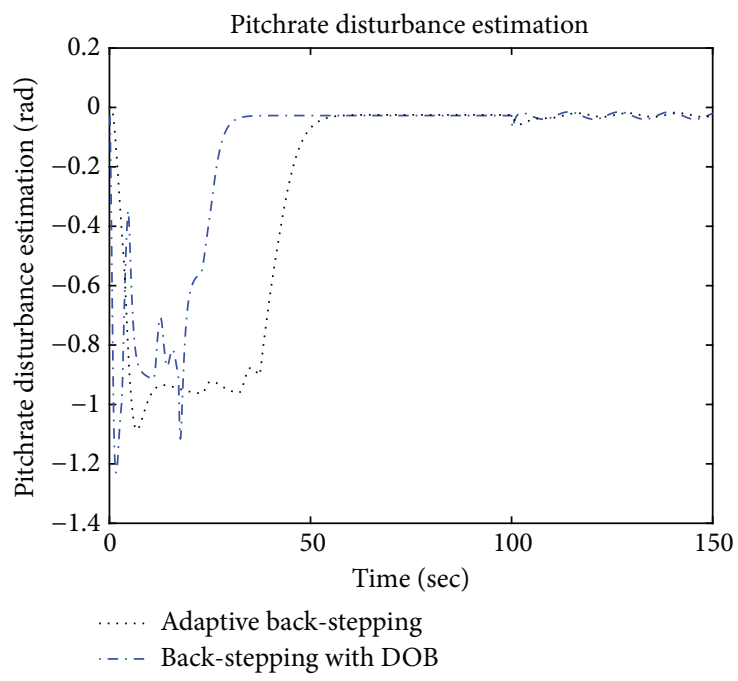

(e)

Figure 4: Lumped disturbances estimated by NDO. 


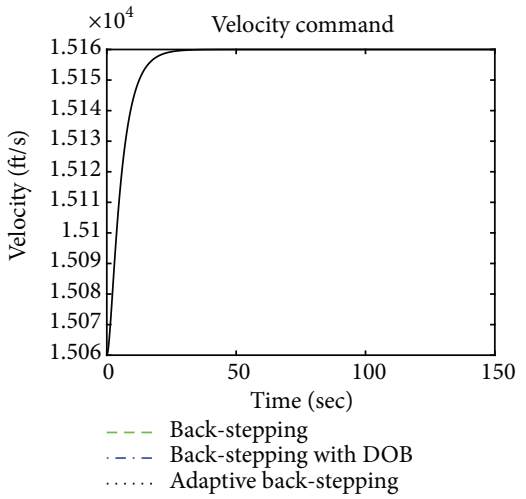

(a)

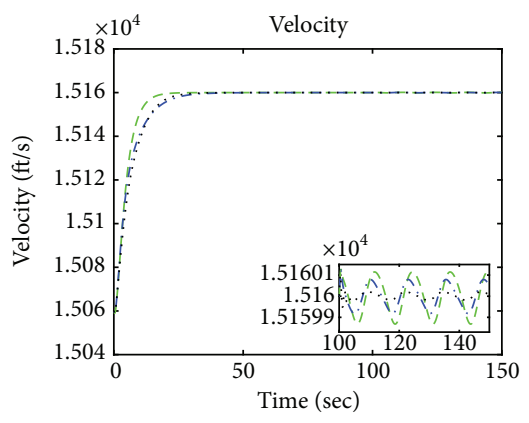

- - - Back-stepping
- - - Back-stepping with DOB
.... Adaptive back-stepping

(c)

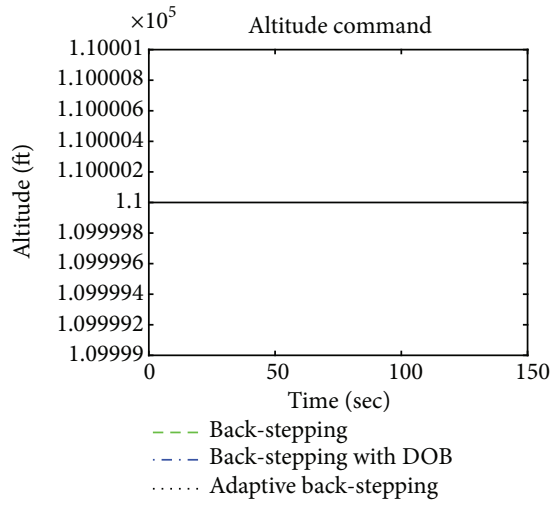

(b)

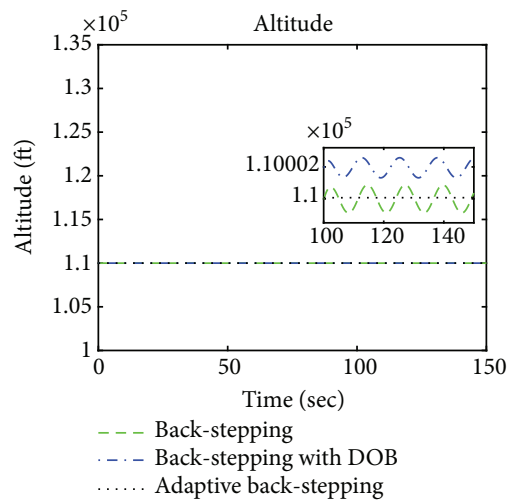

(d)

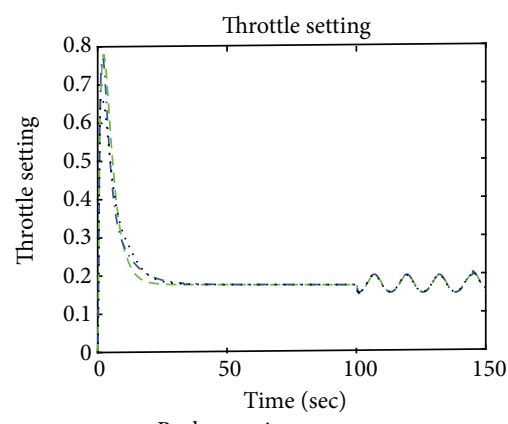

- - Back-stepping

. - . Back-stepping with DOB

..... Adaptive back-stepping

(e)

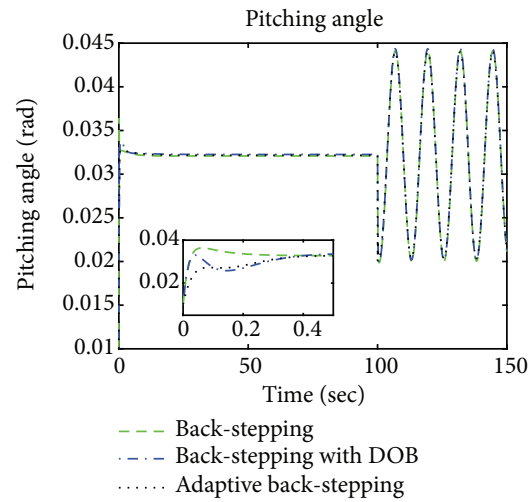

(h)

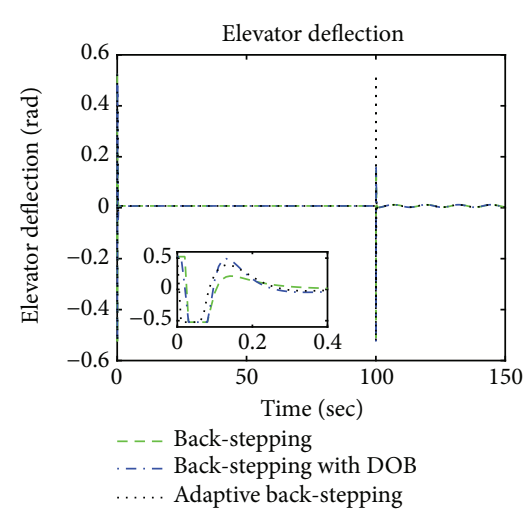

(f)

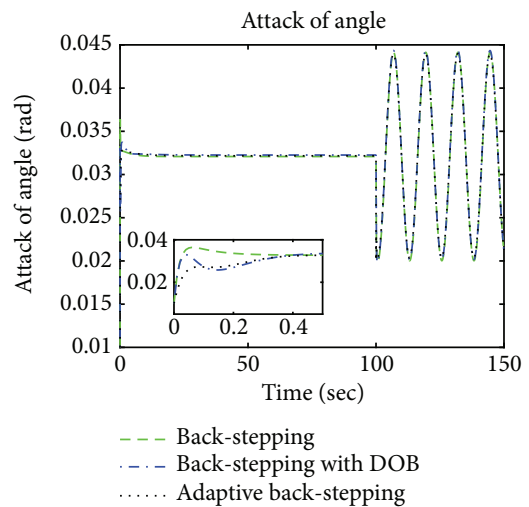

(i)

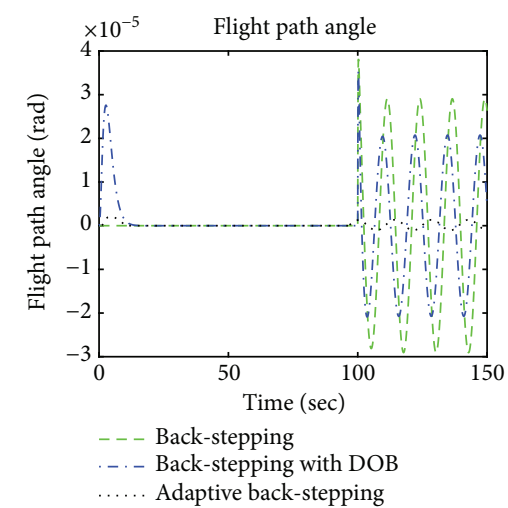

(g)

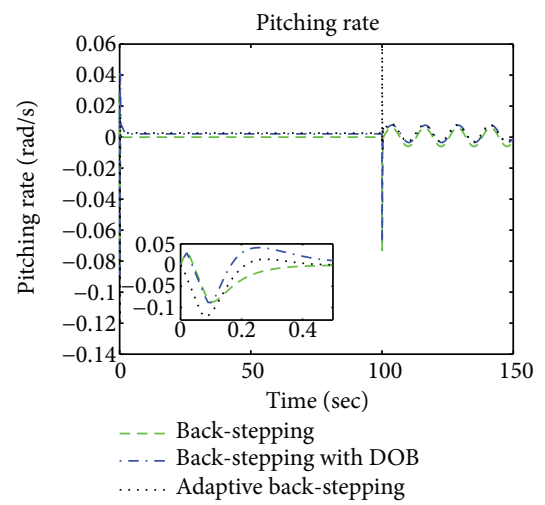

(j)

Figure 5: Velocity command, response, and controller. The uncertainty terms and external disturbances are added into the system. 

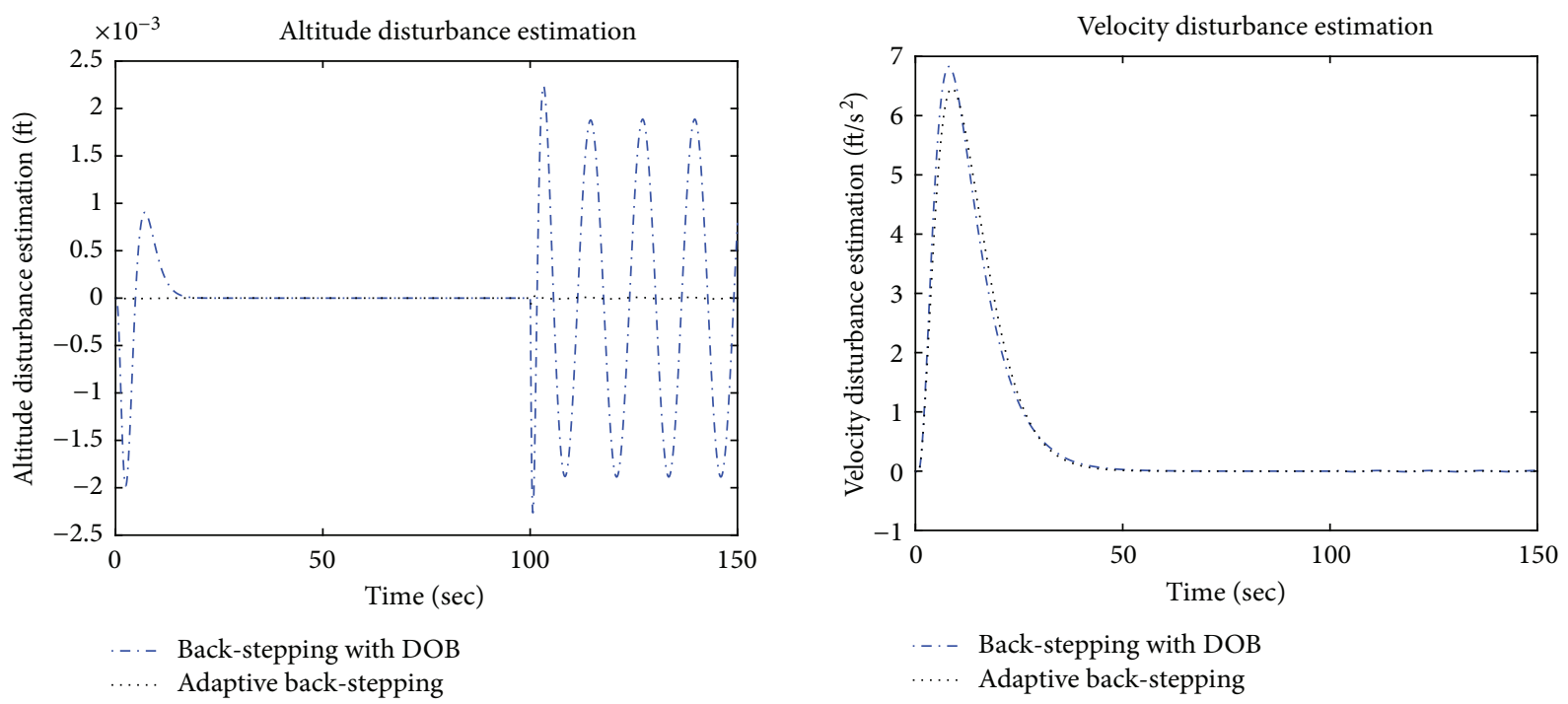

(a)
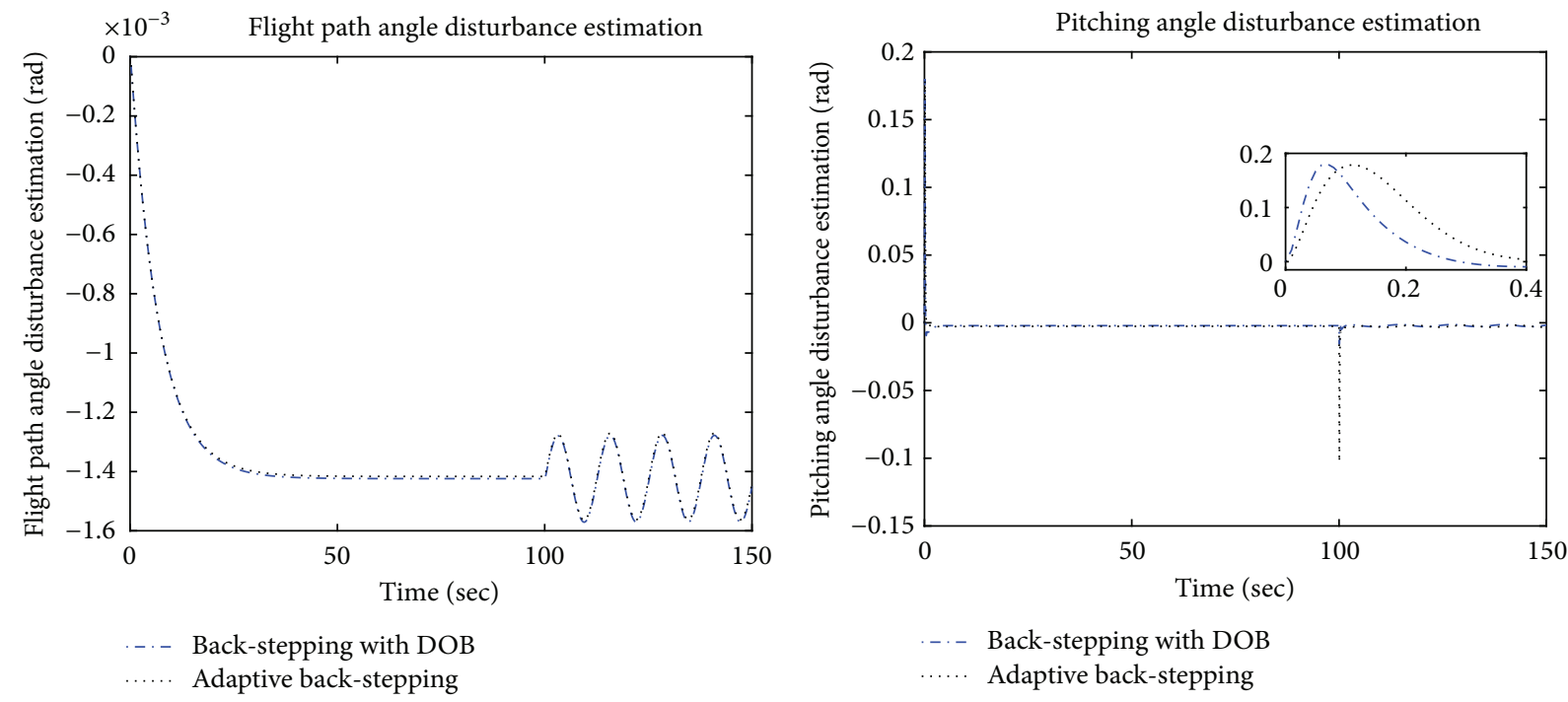

(c)

(d)

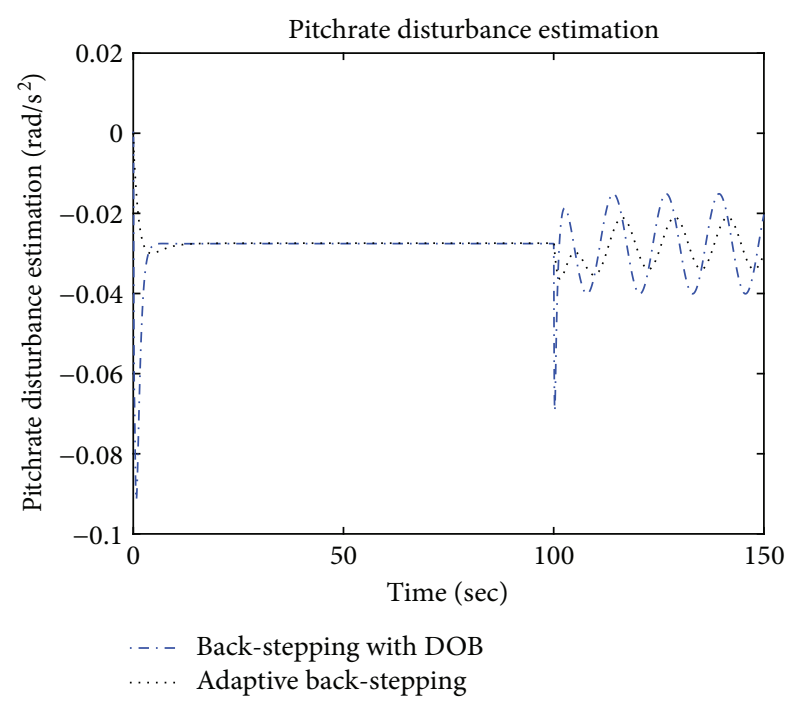

(e)

FIgURE 6: Lumped disturbance estimated by NDO. 


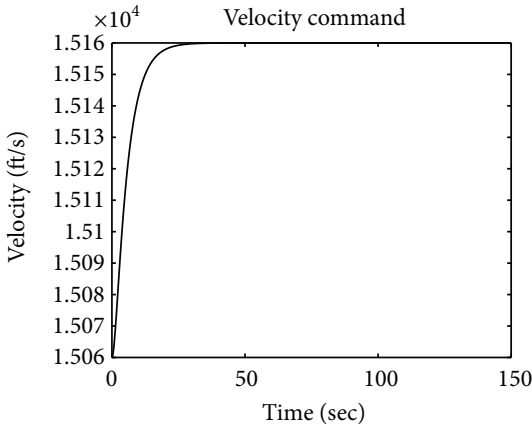

(a)

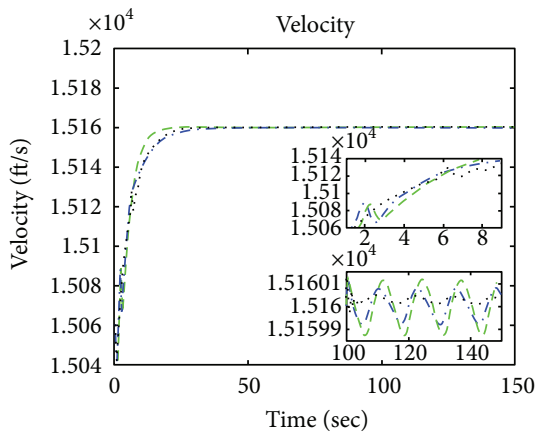

- - - Back-stepping

-. . . Back-stepping with DOB

..... Adaptive back-stepping

(c)

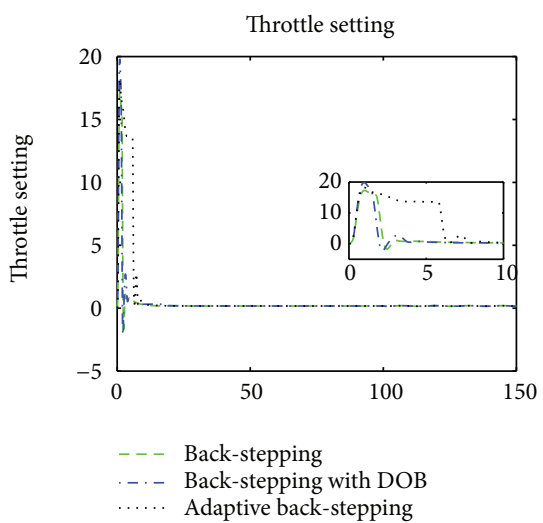

(e)

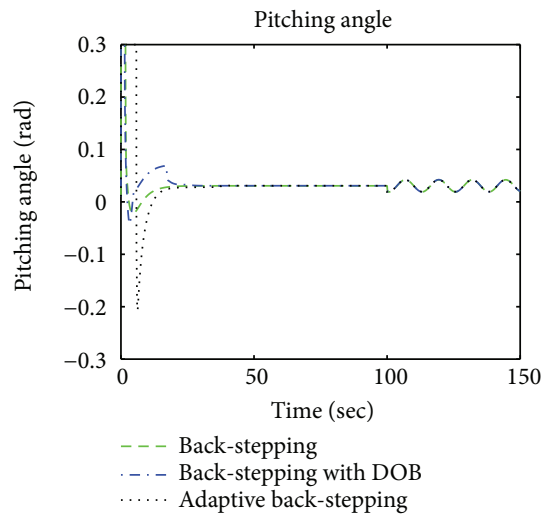

(h)

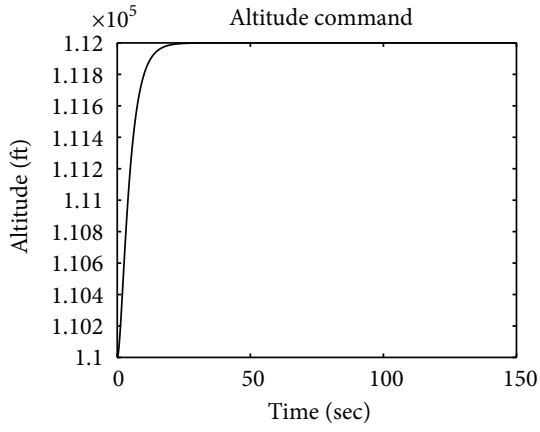

(b)

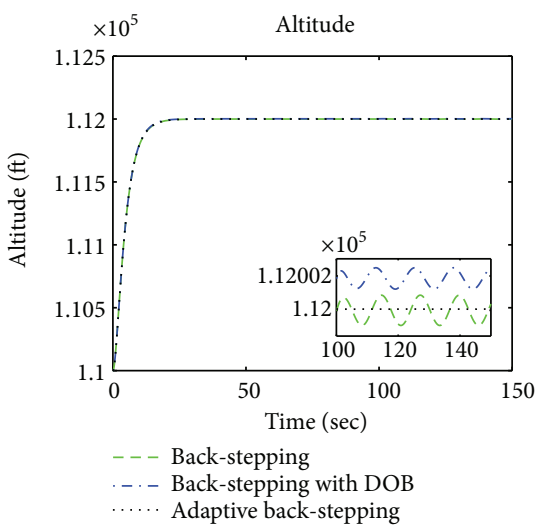

(d)$$
50
$$

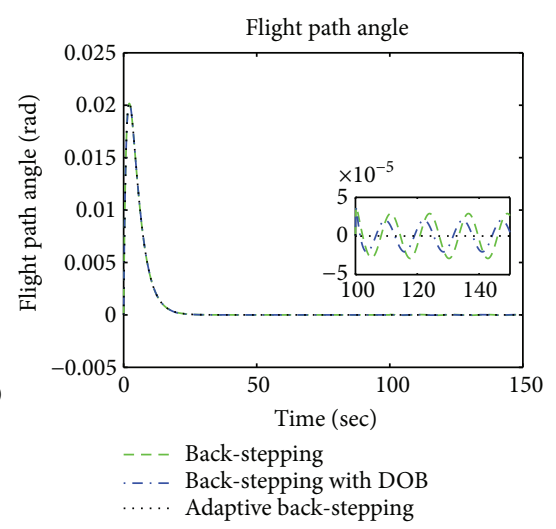

(g)

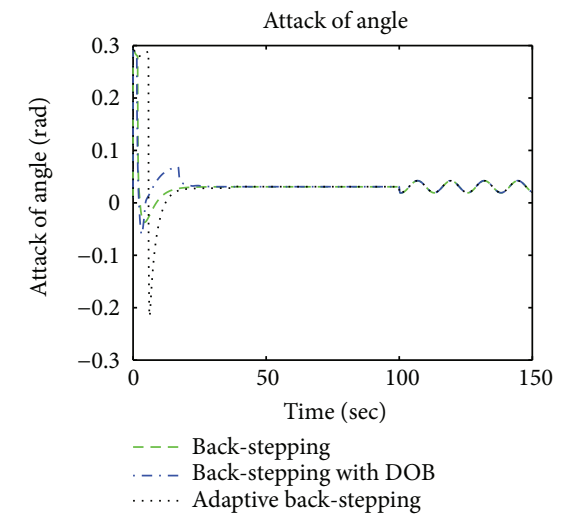

(i)

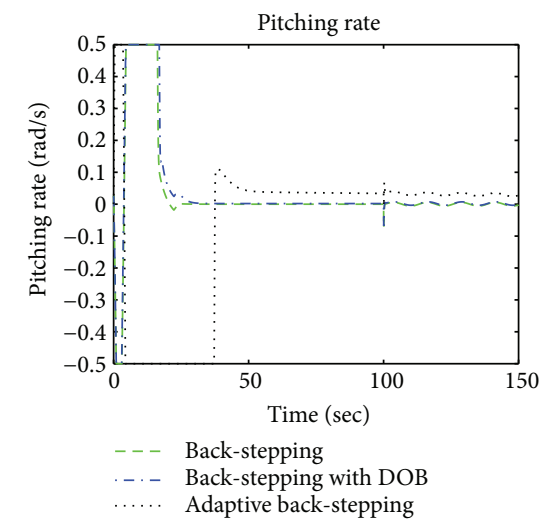

(j)

FIGURE 7: Command, response, and controller. The uncertainty terms and external disturbances are added into the system. 


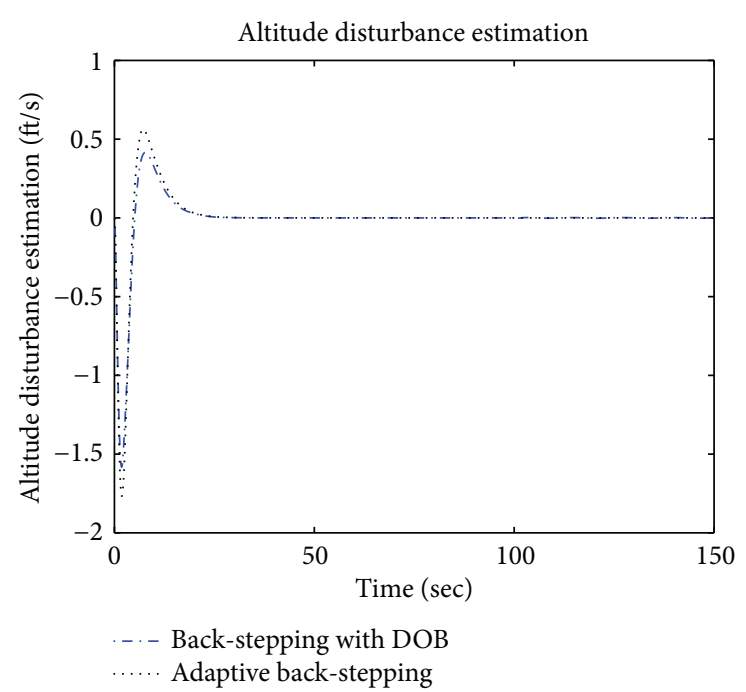

(a)

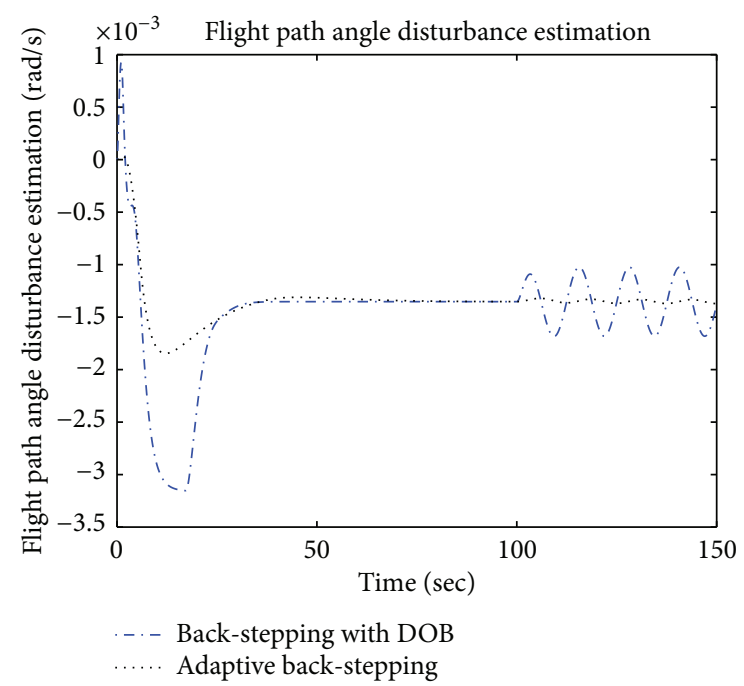

(c)

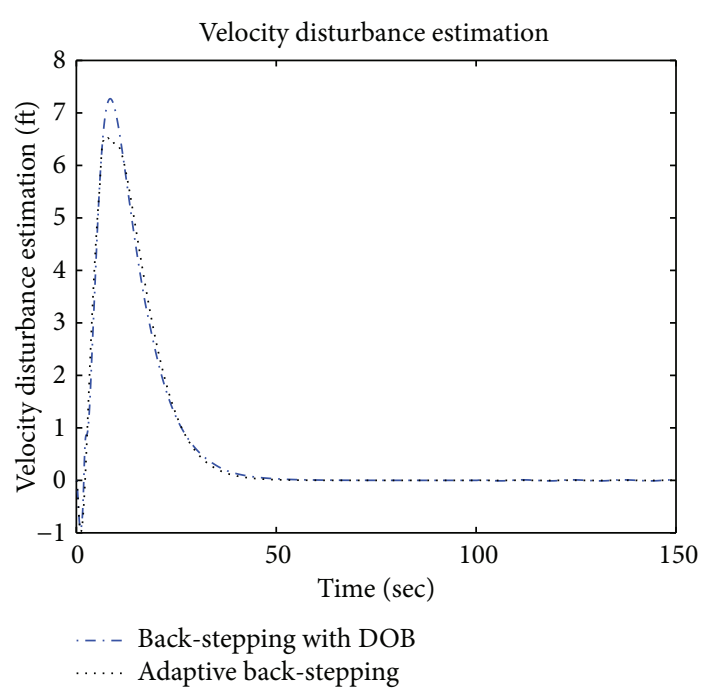

(b)

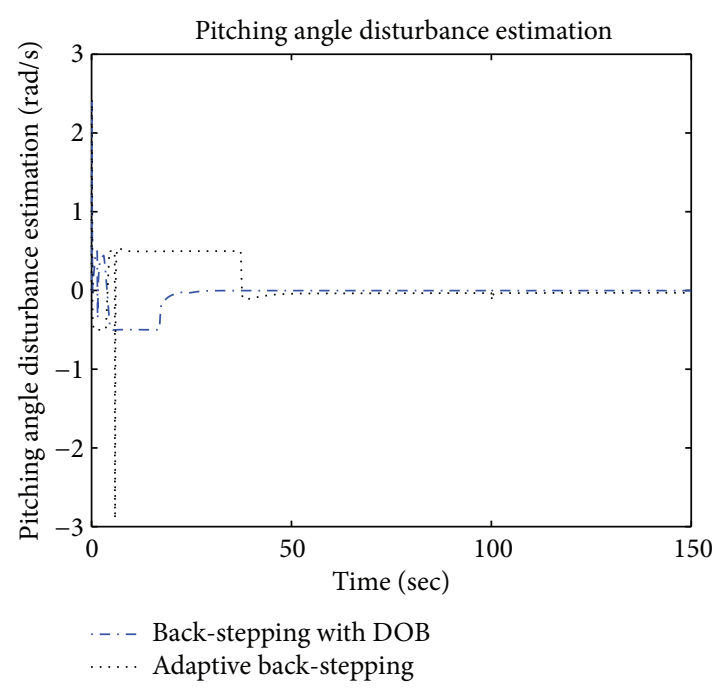

(d)

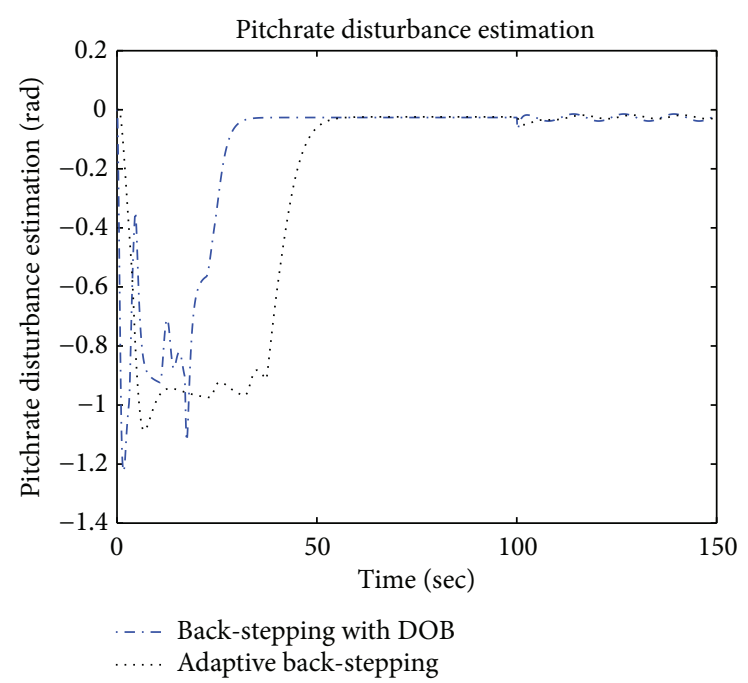

(e)

FIGURE 8: Lumped disturbance estimated by nonlinear disturbance observer. 


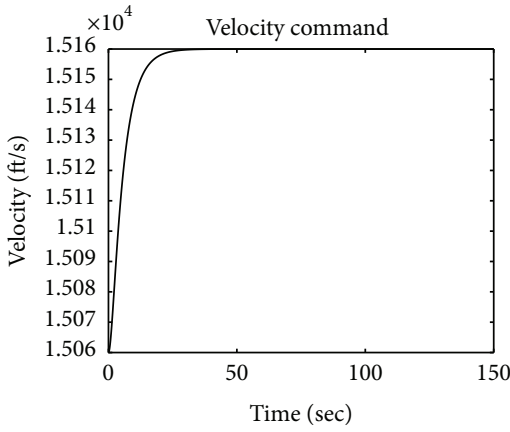

(a)

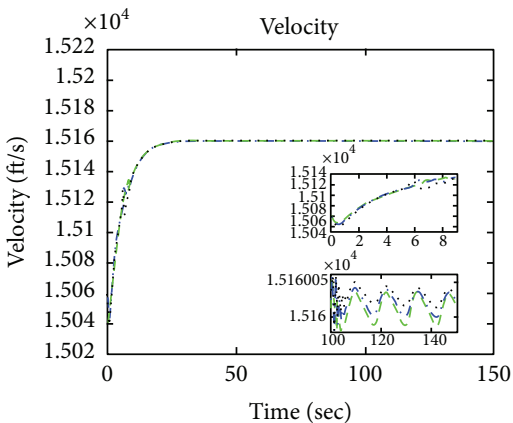

$$
\begin{aligned}
--K & =0.8 \\
--K & =1.0 \\
\cdots-K & =1.2
\end{aligned}
$$

(c)

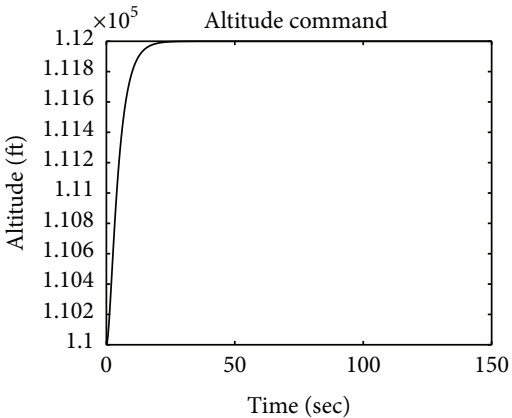

(b)

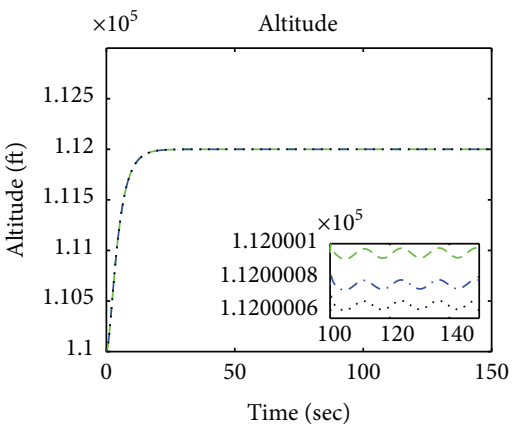

$--K=0.8$

$--K=1.0$

… $K=1.2$

(d)

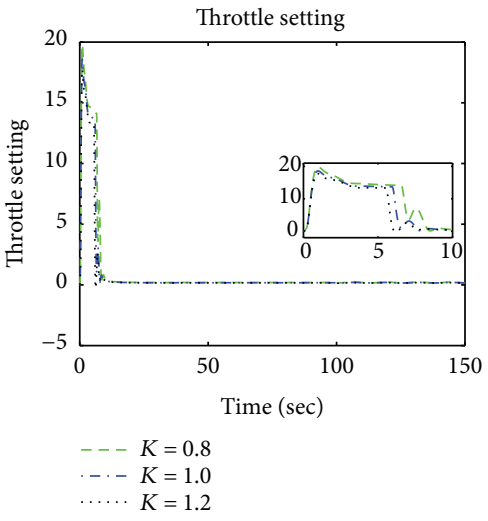

(e)

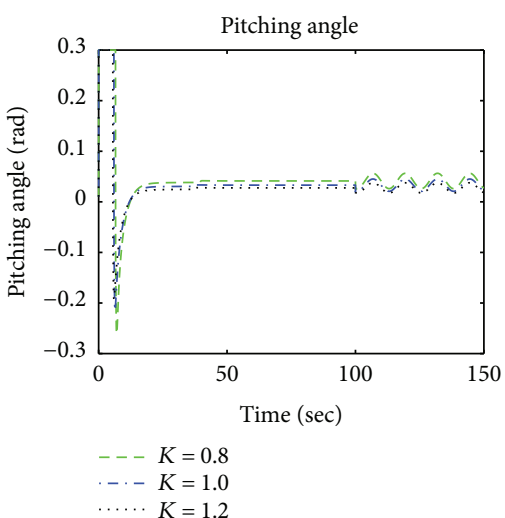

(h)

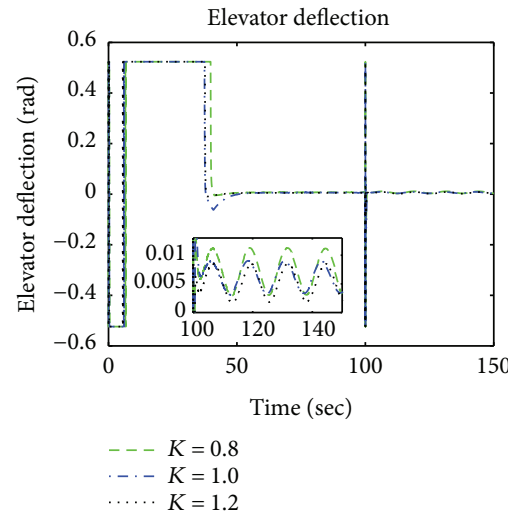

(f)

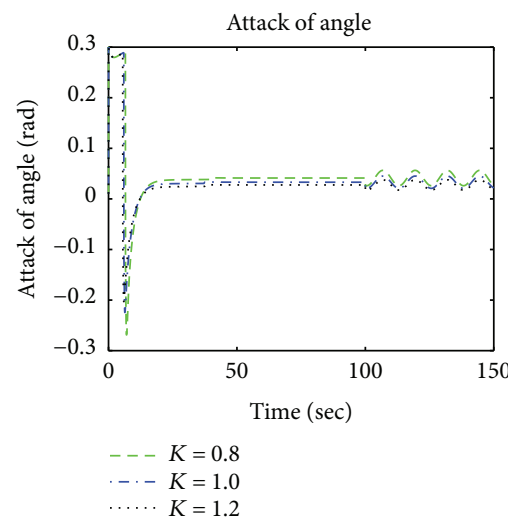

(i)

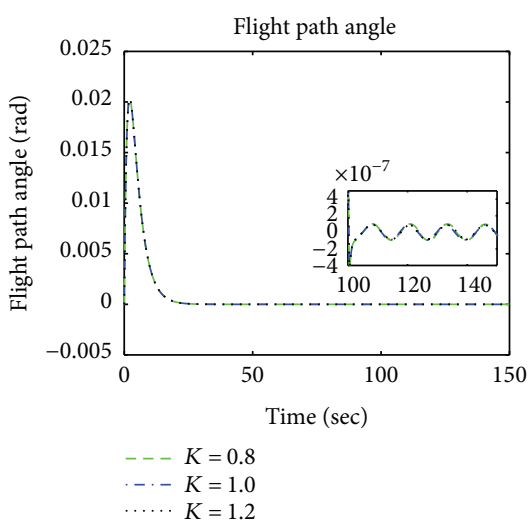

(g)

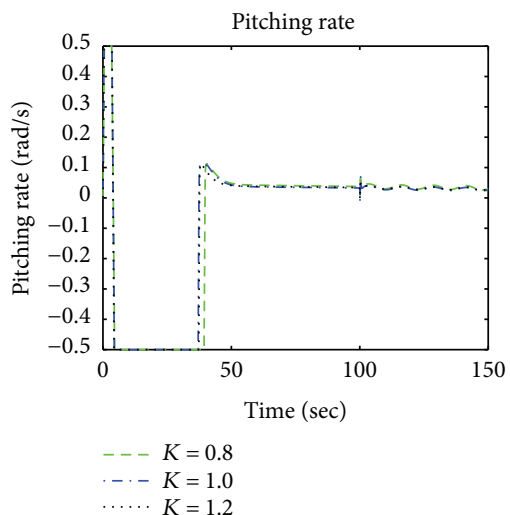

(j)

Figure 9: Altitude command, response, and controller. The uncertainty terms and external disturbance are added into the model. 


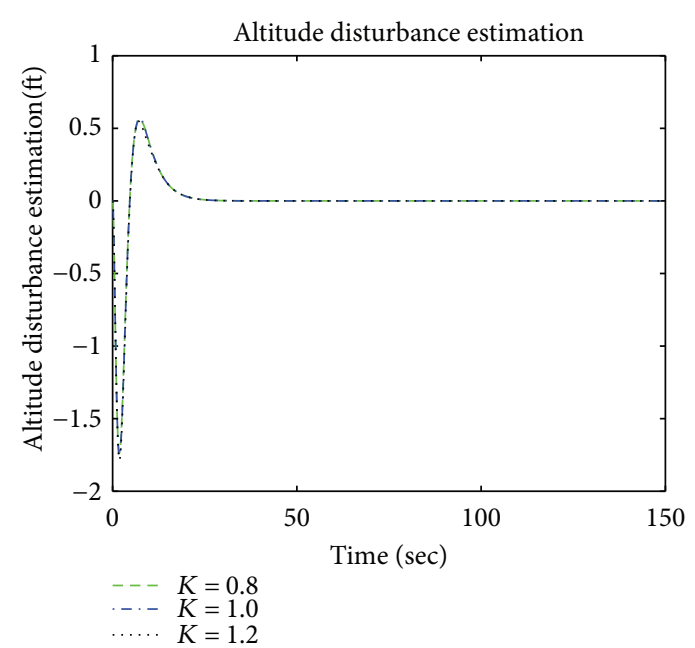

(a)

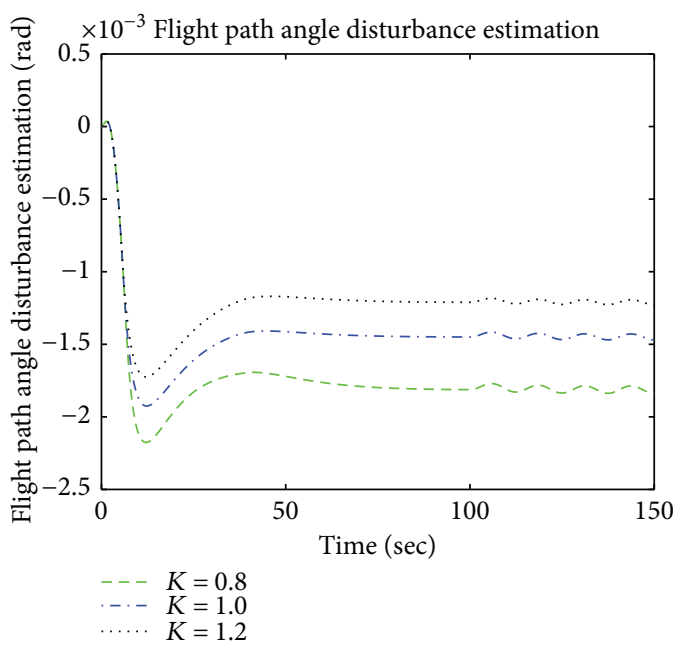

(c)

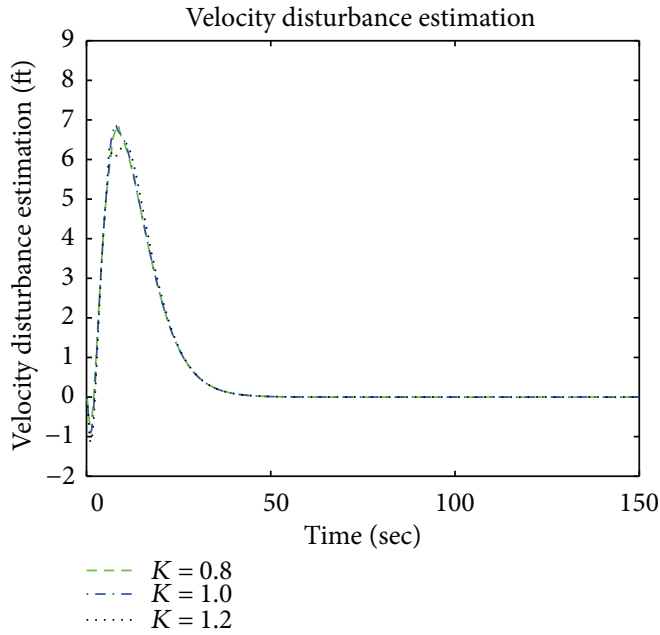

(b)

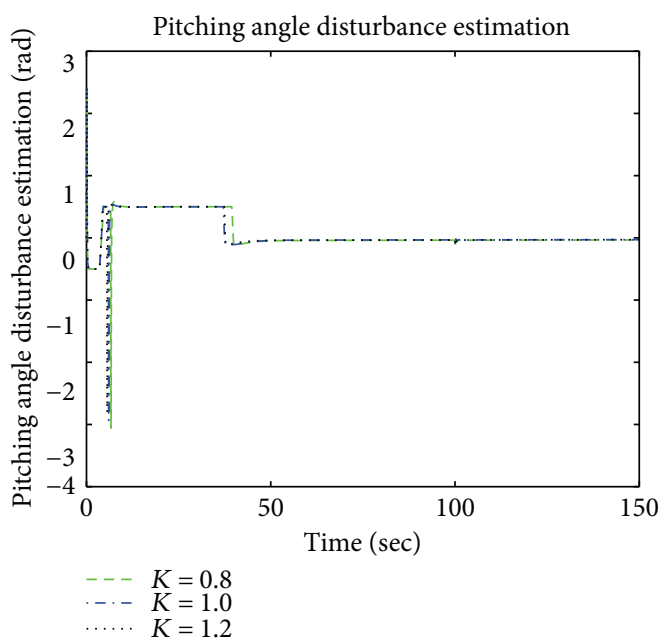

(d)

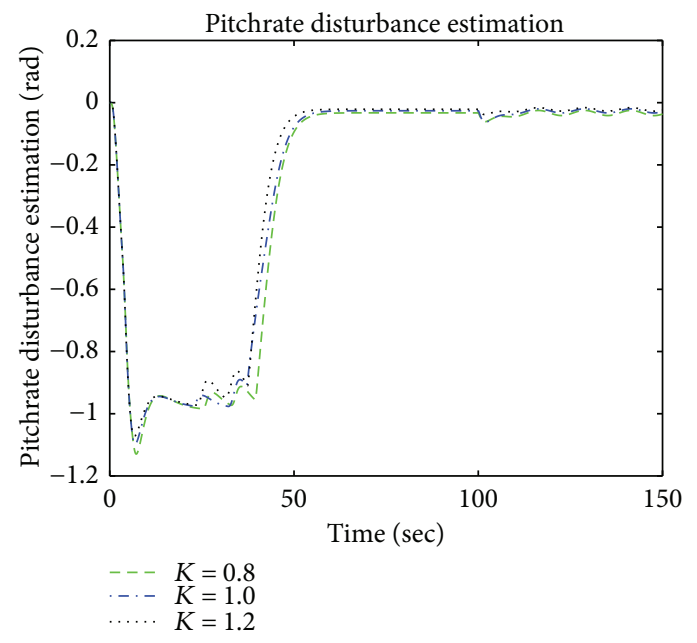

(e)

FIGURE 10: Lumped disturbance estimated by nonlinear disturbance observer. The parameters deflection, uncertainty terms, and external disturbance are added into the model. 
Case 1. The square wave is adopted to prove the effectiveness of controller. The uncertainties are added into this system. The simulation results are shown in Figures 1 and 2.

It is obtained from Figure 1 that the velocity can track the given command well while the altitude is stable. Meanwhile, as shown in Figure 2, the altitude can track the reference command well and the velocity is stable.

Case 2. The proposed controller is compared to the backstepping method [11] and back-stepping with NDO [29]. The external disturbances are added into the model at $t=$ 150 s. The simulation results are shown in Figures 3-8.

The performance of the controller is proved with the existence of uncertainties and external disturbances. At $t=$ $150 \mathrm{~s}$, the external disturbances are taken into the system. Compared with other methods mentioned in Case 2, the performance of GHV under the proposed controller is better.

Case 3. In order to verify the effectiveness of the controller against parameter perturbation, the coefficients of deflection are chosen as $K=0.8, K=1.0$, and $K=1.2$ while the external disturbances are taken at $t=150 \mathrm{~s}$. The simulation results are shown in Figures 9 and 10.

The system under the proposed controller exhibits good performance against both positive and negative parameter perturbation.

\section{Conclusion}

A new adaptive sliding mode control method combined with the nonlinear disturbance observer is proposed to solve the tracking problem for the longitudinal model of a GHV. The compute explosion problem is solved by utilizing the new adaptive control algorithm. In addition, the proposed controller for a GHV model has achieved favorable results in terms of robustness without the cost of sacrificing the nominal control performance. Finally, the performance of the proposed control algorithm has been demonstrated by simulation results.

\section{Conflicts of Interest}

The authors declare that they have no conflicts of interest.

\section{Acknowledgments}

This work was supported by the National Natural Science Foundation of China (no. 61703339).

\section{References}

[1] B. Fidan, M. Mirmirani, and P. Ioannou, "Flight dynamics and control of air-breathing hypersonic vehicles: review and new directions," 12th AIAA International Space Planes and Hypersonic Systems and Technologies, vol. 7081, 2003.

[2] M. A. Bolender and D. B. Doman, "Nonlinear longitudinal dynamical model of an air-breathing hypersonic vehicle," Journal of Spacecraft and Rockets, vol. 44, no. 2, pp. 374-387, 2007.
[3] H. Xu, M. D. Mirmirani, and P. A. Ioannou, "Adaptive sliding mode control design for a hypersonic flight vehicle," Journal of Guidance, Control, and Dynamics, vol. 27, no. 5, pp. 829-838, 2004.

[4] H. Buschek and A. J. Calise, "Uncertainty modeling and fixed-order controller design for a hypersonic vehicle model," Journal of Guidance, Control, and Dynamics, vol. 20, no. 1, pp. 42-48, 1997.

[5] X. Jiao, J. Jiang, H. Xu, and H. Meng, "Robust controller of hypersonic aircraft based on grey prediction sliding mode control," Journal of Grey System, vol. 28, no. 3, 2016.

[6] F. Wang, Q. Zong, Q. Dong, and B. Tian, "Disturbance observer-based sliding mode backstepping control for a reentry vehicle with input constraint and external disturbance," Transactions of the Institute of Measurement and Control, vol. 38, no. 2, pp. 165-181, 2016.

[7] H. Sun, S. Li, and C. Sun, "Finite time integral sliding mode control of hypersonic vehicles," Nonlinear Dynamics, vol. 73, no. 1-2, pp. 229-244, 2013.

[8] J. Wang, Q. Zong, R. Su, and B. Tian, "Continuous high order sliding mode controller design for a flexible air-breathing hypersonic vehicle," ISA Transactions, vol. 53, no. 3, pp. 690-698, 2014.

[9] X. Bu, X. Wu, J. Huang, Z. Ma, and R. Zhang, "Minimal-learning-parameter based simplified adaptive neural back-stepping control of flexible air-breathing hypersonic vehicles without virtual controllers," Neurocomputing, vol. 175, pp. 816-825, 2016.

[10] B. Xu and Y. Zhang, "Neural discrete back-stepping control of hypersonic flight vehicle with equivalent prediction model," Neurocomputing, vol. 154, pp. 337-346, 2015.

[11] W. Peng, L. Peng, and L. Ming, "Backstepping control for longitudinal dynamics of hypersonic flight vehicle," in Proceedings of 2014 IEEE Chinese Guidance, Navigation and Control Conference, pp. 1051-1055, Yantai, China, August 2014.

[12] C. Chen, K. Zhu, G. Ma, and Y. Lv, "Adaptive backstepping control for reentry attitude of near space hypersonic vehicle with input saturation," in 2016 35th Chinese Control Conference (CCC), pp. 10742-10747, Chengdu, China, July 2016.

[13] J. Wang, J. Wang, P. Yang, K. Liu, X. Yu, and J. Hu, “Adaptive sliding mode control for a flexible hypersonic vehicle with actuator saturation," in 2016 35th Chinese Control Conference (CCC), pp. 647-651, Chengdu, China, July 2016.

[14] R. Zhai, R. Qi, and B. Jiang, "Adaptive sliding mode faulttolerant control for hypersonic aircraft using RBF neural networks," in Decision Conference (CCDC), pp. 1879-1884, Yinchuan, China, May 2016.

[15] J. Fu, L. Wang, M. Chen, and S. Chang, "Robust adaptive attitude control for airbreathing hypersonic vehicle with attitude constraints and propulsive disturbance," Mathematical Problems in Engineering, vol. 2015, Article ID 293480, 11 pages, 2015.

[16] B. Xu, Y. Guo, Y. Yuan, Y. Fan, and D. Wang, "Fault-tolerant control using command-filtered adaptive back-stepping technique: application to hypersonic longitudinal flight dynamics," International Journal of Adaptive Control and Signal Processing, vol. 30, no. 4, pp. 553-577, 2015.

[17] Y. Zhang, R. Li, T. Xue, and Z. Lei, "Exponential sliding mode tracking control via back-stepping approach for a hypersonic vehicle with mismatched uncertainty," Journal of the Franklin Institute, vol. 353, no. 10, pp. 2319-2343, 2016. 
[18] Q. Zong, Y. Ji, F. Zeng, and H. Liu, "Output feedback back-stepping control for a generic hypersonic vehicle via small-gain theorem," Aerospace Science and Technology, vol. 23, no. 1, pp. 409-417, 2012.

[19] L. Fiorentini, A. Serrani, M. A. Bolender, and D. B. Doman, "Nonlinear robust adaptive control of flexible air-breathing hypersonic vehicles," Journal of Guidance, Control, and Dynamics, vol. 32, no. 2, pp. 402-417, 2009.

[20] T. E. Gibson, L. G. Crespo, and A. M. Annaswamy, "Adaptive control of hypersonic vehicles in the presence of modeling uncertainties," in 2009 American Control Conference, pp. 3178-3183, St. Louis, MO, USA, June 2009.

[21] H. Sun, L. Hou, and Y. Li, "Disturbance observer based dynamic surface tracking control for a class of uncertain nonlinear systems with mismatched disturbances," in 2016 12th World Congress on Intelligent Control and Automation (WCICA), pp. 605-610, Guilin, China, June 2016.

[22] W. H. Chen, "Nonlinear disturbance observer-enhanced dynamic inversion control of missiles," Journal of Guidance, Control, and Dynamics, vol. 26, no. 1, pp. 161-166, 2003.

[23] S. Li, J. Yang, W. H. Chen, and X. Chen, "Disturbance observer-based control: methods and applications," International Standard Book Number, vol. 13, 2014.

[24] J. Yang, S. Li, C. Sun, and L. Guo, "Nonlinear-disturbanceobserver-based robust flight control for airbreathing hypersonic vehicles," IEEE Transactions on Aerospace and Electronic Systems, vol. 49, no. 2, pp. 1263-1275, 2013.

[25] C. E. Hall and Y. B. Shtessel, "Sliding mode disturbance observer-based control for a reusable launch vehicle," Journal of Guidance, Control, and Dynamics, vol. 29, no. 6, pp. 13151328, 2006.

[26] T. Y. Zhang, J. Zhou, and J. G. Guo, "Design of predictive controller for hypersonic vehicles based on disturbance observer," Acta Aeronautica Et Astronautica Sinica, vol. 35, no. 1, pp. 215-222, 2014.

[27] Y. Liu, J. Guo, and J. Zhou, "Hypersonic vehicle attitude control based on new fast terminal sliding mode," Acta Aeronautica Et Astronautica Sinica, vol. 36, no. 7, pp. 2372-2380, 2015.

[28] B. Tian, L. Yin, and H. Wang, "Finite-time reentry attitude control based on adaptive multivariable disturbance compensation," IEEE Transactions on Industrial Electronics, vol. 62, no. 9, pp. 5889-5898, 2015.

[29] Z. Yang, B. Meng, and H. Sun, "A new kind of nonlinear disturbance observer for nonlinear systems with applications to cruise control of air-breathing hypersonic vehicles," International Journal of Control, vol. 90, no. 9, pp. 1935-1950, 2016. 


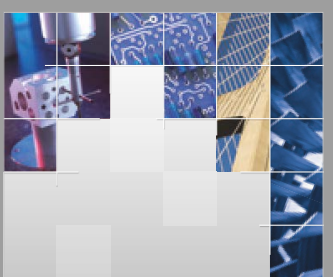

\section{Enfincering}
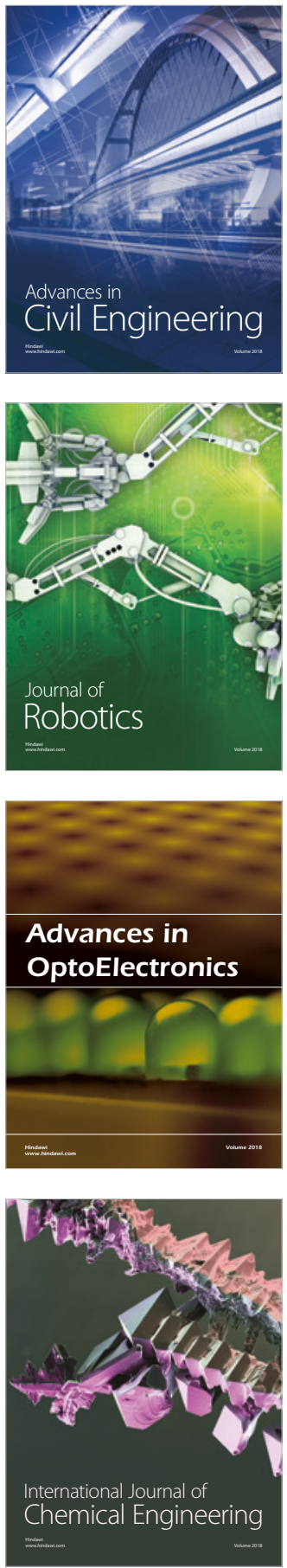

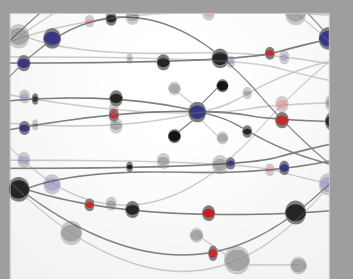

\section{Rotating \\ Machinery}

The Scientific World Journal

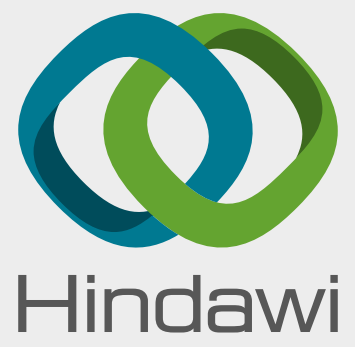

Submit your manuscripts at

www.hindawi.com
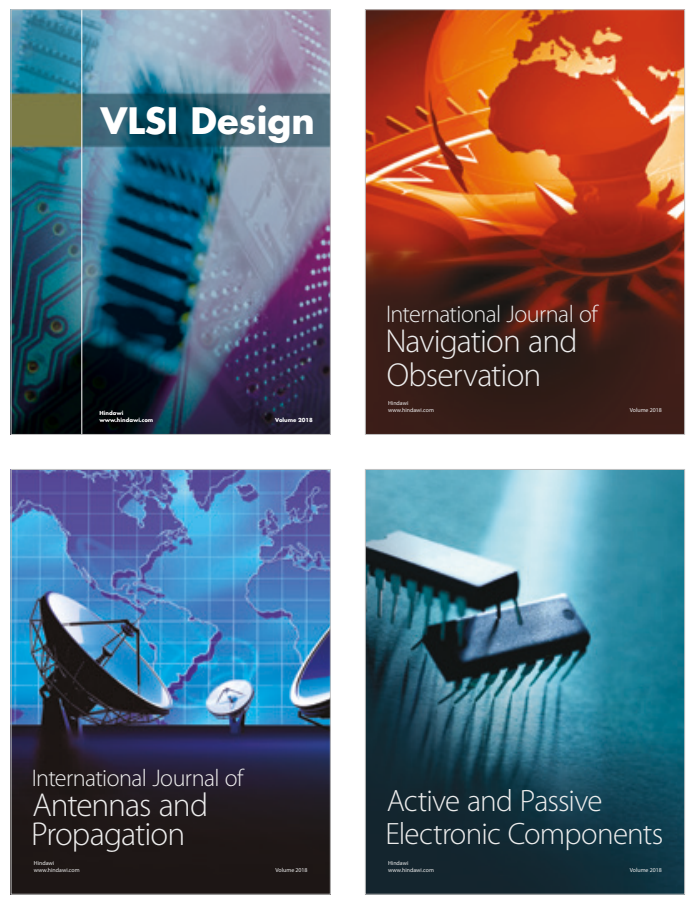
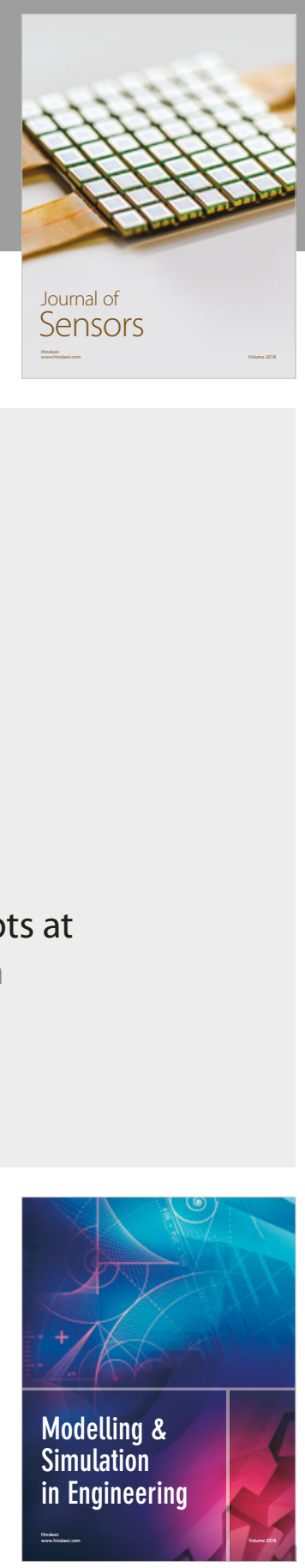

\section{Advances \\ Multimedia}
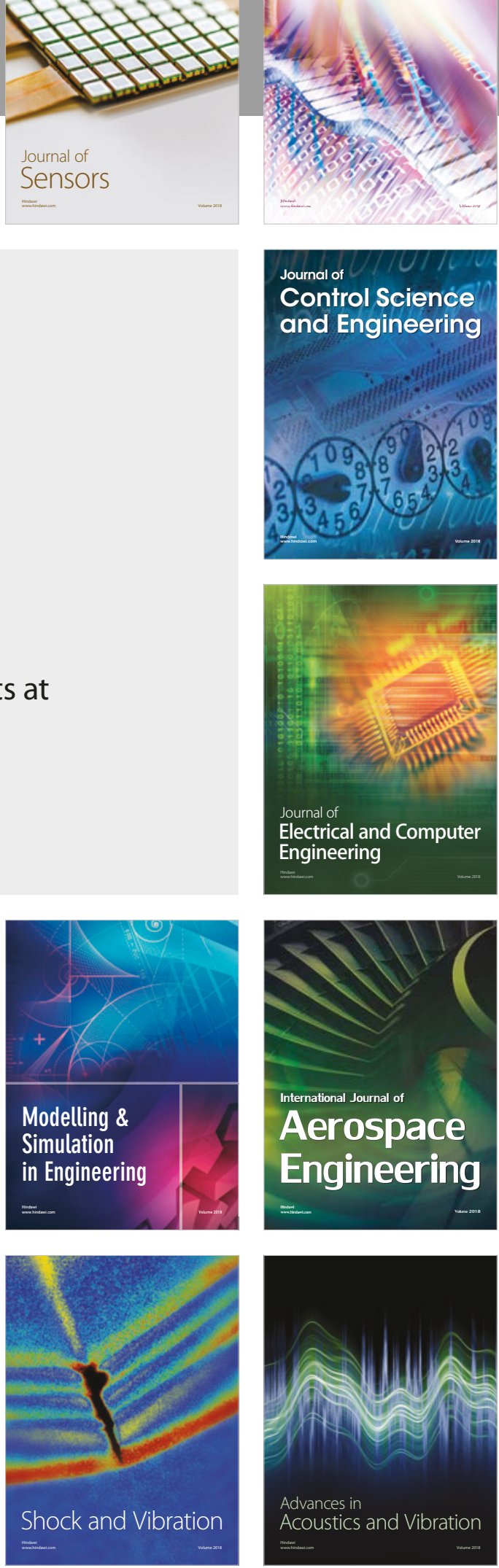